\title{
A comprehensive analysis of electric dipole moment constraints on CP-violating phases in the MSSM
}

\author{
Yingchuan Li, ${ }^{a}$ Stefano Profumo ${ }^{b}$ and Michael Ramsey-Musolf ${ }^{a, c}$ \\ Department of Physics, University of Wisconsin, \\ Madison, Wisconsin 53706 U.S.A. \\ Department of Physics and Santa Cruz Institute for Particle Physics, \\ University of California, 1156 High St., Santa Cruz, CA 95064, U.S.A. \\ Kellogg Radiation Laboratory, California Institute of Technology, \\ Pasadena, CA 91125 U.S.A. \\ E-mail: yli@physics.wisc.edu, profumo@scipp.ucsc.edu, \\ mjrm@physics.wisc.edu
}

ABSTRACT: We analyze the constraints placed on individual CP-violating phases in the minimal supersymmetric extension of the Standard Model (MSSM) by current experimental bounds on the electric dipole moments (EDMs) of the neutron, Thallium, and Mercury atoms. We identify the four CP-violating phases that are individually highly constrained by current EDM bounds, and we explore how these phases and correlations among them are constrained by current EDM limits. We also analyze the prospective implications of the next generation of EDM experiments. We point out that all other CP-violating phases in the MSSM are not nearly as tightly constrained by limits on the size of EDMs. We emphasize that a rich set of phenomenological consequences is potentially associated with these generically large EDM-allowed phases, ranging from B physics, electroweak baryogenesis, and signals of CP-violation at the CERN Large Hadron Collider and at future linear colliders. Our numerical study takes into account the complete set of contributions from one- and two-loop EDMs of the electron and quarks, one- and two-loop ChromoEDMs of quarks, the Weinberg 3-gluon operator, and dominant 4-fermion CP-odd operator contributions, including contributions which are both included and not included yet in the CPsuperH2.0 package. We also introduce an open-source numerical package, 2LEDM, which provides the complete set of two-loop electroweak diagrams contributing to the electric dipole moments of leptons and quarks.

KeYwords: Supersymmetry Phenomenology

ARXIV EPRINT: 1006.1440 


\section{Contents}

1 Introduction 1

2 CP-violating phases in MSSM and the setup for analysis 4

3 Detailed analysis $\quad 6$

3.1 Phases in the higgsino-gaugino sector: $\phi_{1}, \phi_{2}$, and $\phi_{3} \quad 7$

3.2 Phases in the sfermion sector: $\phi_{e}, \phi_{u}, \phi_{d}, \phi_{\mu}, \phi_{c}, \phi_{s}, \phi_{t}, \phi_{b}, \phi_{\tau} \quad 10$

3.3 Correlated constraints on the most strongly constrained phases $\phi_{2}, \phi_{3}$ and $\phi_{u, d} 13$

3.4 Implication of loosely constrained phases on CP-violating and CP-conserv$\begin{array}{ll}\text { ing phenomenologies } & 18\end{array}$

4 Conclusions

\section{Introduction}

The minimal supersymmetric extension of the standard model (MSSM) features a large number of additional CP-violating sources compared to the SM, yielding an extremely rich array of possible phenomenological consequences $[1,2]$. To mention a few, the new sources of $\mathrm{CP}$ violation can participate in the generation of the baryon asymmetry of the universe in the context of supersymmetric electroweak baryogenesis [3-11]. They can generate observable CP asymmetries at the CERN Large Hadron Collider (LHC) [12-14] as well as at future linear colliders [15-21]. They could manifest themselves in B physics (see ref. [22-26] and the recent results from the Tevatron D0 Collaboration [27] and CDF Collaboration(for preliminary $2010 \mathrm{CDF}$ results, see e.g. [28])). In general, CP-violating phases induce rather large contributions, compared to current experimental sensitivity, to the electric dipole moment (EDM) of the neutron and of atoms [29]. A large CP-violating phase could also have an effect on many CP-conserving observables such as the mass spectrum, the production rate and decay branching ratios of SUSY particles, especially the lightest neutralino as a dark matter particle (including direct and indirect detection rates, and the relic density) [30-33] and Supersymmetric Higgs scalars [34, 35].

All these potentially interesting observable signatures are however highly constrained by the fact that no permanent EDM has ever been experimentally observed. The current most stringent bounds are on the EDMs of the neutron [36], and on that of Thallium [37] and Mercury [38] atoms. ${ }^{1}$ Specifically, the current experimental constraints on the afore-

\footnotetext{
${ }^{1}$ For recent reviews on EDM searches and their implications for the MSSM, see, e.g. refs. [2, 29, 39].
} 
mentioned quantities read:

$$
\begin{aligned}
\left|d_{\mathrm{n}}\right| & <2.9 \times 10^{-26} \text { e cm }(90 \% \text { C.L. }), \\
\left|d_{\mathrm{Tl}}\right| & <9.0 \times 10^{-25} \text { e cm }(90 \% \text { C.L. }), \\
\left|d_{\mathrm{Hg}}\right| & <3.1 \times 10^{-29} \text { e cm }(95 \% \text { C.L. }) .
\end{aligned}
$$

Signatures of CP-violation compatible with the bounds described above have so far been discussed in the context of scenarios where one avoids EDM bounds and keeps relatively large phases with light super-particle masses via one or more cancelations among the various terms contributing to the EDMs [16, 39-45]. This "cancellation" scenario occurs typically in some very fine-tuned region ${ }^{2}$ of the MSSM parameter space. The goal of the present analysis is instead to examine in detail, beyond the possibility of cancelations between different CP-violating sources, ${ }^{3}$ how each individual phase is constrained by current EDM bounds and to study under which conditions large phases are phenomenologically allowed while also keeping the relevant mass scales relatively light and thus phenomenologically interesting. Instead of imposing any universality condition as in the supergravity (SUGRA) model, we entertain here the possibility of general non-universal soft terms (as is the case in many string-inspired models, see e.g. [47-49]), and thus keep all the soft terms in MSSM as independent variables. One of our main results is that among all MSSM $\mathrm{CP}$-violating phases, only four are in fact strongly constrained by current EDM bounds, and we study the correlations among them.

Part of our numerical study is based on the CPsuperH2.0 package [50,51], which includes the complete contributions from one-loop supersymmetric contributions to the EDMs of the electron and of quarks, one-loop and two-loop Chromo-EDMs of quarks [4143, 52-55], 6-dimensional 3-gluon Weinberg operator [56-58], and dominant contributions to 4-fermion CP-odd operators [59], but only a subset of the contributions of two-loop EDMs of electron and quarks [52-55]. There exist additional Higgs exchange-mediated chargino-neutralino 2-loop contributions to electron and quark EDMs that can become dominant in the limit of heavy sfermions [60] and that are not included in CPsuperH2.0 package. We here take into account these contributions as well, therefore featuring the dominant 1-loop and 2-loop contributions to all the 6-dimensional CP-odd operators that generate EDMs of the neutron and of Thallium and Mercury atoms. ${ }^{4}$ The numerical code where all these new contributions are collected is called 2LEDM, and is currently available from the authors upon request. The 2LEDM code currently includes an interface to the FeynHiggs ${ }^{5}$ package (version 2.6.5) [61]. We plan in the near future to set up a webpage for easier download of 2LEDM and of related tutorials and to include an interface to the CPsuperH2.0 package.

\footnotetext{
${ }^{2}$ It is estimated in ref. [46] that a minimal degree of fine-tuning at the level of $10^{-2}$ is needed.

${ }^{3}$ There are still cancelations among different contributions to EDMs from the same source in some region of parameter space. We emphasize that our main focus is on the general trend of constraints without cancelation, and always point it out when we encounter such cancelation region.

${ }^{4}$ The four fermion operators in the MSSM are technically dimension eight.

${ }^{5}$ http://www.feynhiggs.de/
} 
There remains an order one theoretical uncertainty with neutron and Mercury EDMs. For the neutron EDM, the uncertainty arises from hadronic physics, while for the Mercury EDM the source of uncertainty is associated with (i) atomic physics in extracting the nuclear Schiff moment from $d_{\mathrm{Hg}}$, (ii) the nuclear physics going into extracting T- and Podd pion-nucleon couplings $\bar{g}_{\pi N N}$ from the Schiff moment, and (iii) the hadronic physics in computing the $\bar{g}_{\pi N N}$ in terms of quark Chromo-EDM operator, Weinberg three-gluon operator, and CP-violating four fermion operators. ${ }^{6}$ In utilizing the CPsuperH2.0 package to estimate the relevant EDMs from the EDMs of quarks and leptons we are relying on QCD sum rule computations $[59,62-66]$ of strong interaction matrix elements. For a discussion of the systematic uncertainties in our results for these quantities when different hadronic model approximations are employed we refere the reader to e.g. ref. [39]. We note also that the CPsuperH2.0 code relies on the computations of the nuclear Schiff moment reported in ref. [67] and does not take into account the recent computations of refs. [68, 69]. The latter two computations give an enhanced sensitivity of the nuclear Schiff moment of ${ }^{199} \mathrm{Hg}$ to the isoscalar, T- and P-odd pion-nucleon coupling, $\bar{g}_{\pi}^{(0)}$ as compared to ref. [67]. Moreover, ref. [69] finds that the sensitivity to $\bar{g}_{\pi}^{(1)}$ may be reduced by a factor of three to ten, depending on the type of interaction used.

In general, $\bar{g}_{\pi}^{(j)}$ are dominated by quark Chromo-EDMs rather than the Weinberg three gluon operator (the contribution is suppressed by $m_{q}$ ), while in the MSSM with large $\tan \beta$, contributions from the four fermion operators may be important [29]. The QCD sum rule analysis implies that $\bar{g}_{\pi}^{(0)}$ is five times less sensitive to the sum of up and down quark Chromo-EDMs than $\bar{g}_{\pi}^{(1)}$ is to their difference. Thus, we would only expect the stronger sensitivity of the Mercury Schiff moment to $\bar{g}_{\pi}^{(0)}$ to be important in small corners of the MSSM parameter space where the difference of quark Chromo-EDMs is highly suppressed compared to their average value. The possible suppression in sensitivity to $\bar{g}_{\pi}^{(1)}$ is a potentially more serious issue. Thus, one may need to relax the constraints we obtain on $\mathrm{CP}$-violating phases that are driven by the ${ }^{199} \mathrm{Hg}$ results in light of on-going theoretical nuclear structure developments.

With these caveats in mind, we summarize our main findings here:

(a) A primary impact of the new ${ }^{199} \mathrm{Hg}$ result is to impose significantly more stringent constraints on the relative phase $\phi_{3}$ between the gluino soft supersymmetric-breaking mass and the $\mu$ parameter (see below), while generating a strong correlation between this phase and the phase of the soft-breaking triscalar couplings involving first generation sfermions.

(b) The neutron and Thallium EDM limits have a stronger impact on the relative phase $\phi_{2}$ between the wino soft mass parameter and $\mu$ than does the ${ }^{199} \mathrm{Hg}$ bound, but at present there does not exist any strong correlation between $\phi_{2}$ and other phases.

(c) A future neutron EDM limit that is roughly 100 times stronger than present would both tighten the present correlations between $\phi_{3}$ and the triscalar phases while inducing strong correlations between $\phi_{2}$ and other phases.

\footnotetext{
${ }^{6}$ In the MSSM, the Chromo-EDM operator typically gives the dominant contribution to the $\bar{g}_{\pi N N}$.
} 


\begin{tabular}{|c|c|c|}
\hline CP-violating phases & one-loop contribution & two-loop contribution \\
\hline$\phi_{e, u, d}$ & $d_{u, d, e}^{1-l o p}, \tilde{d}_{u, d}^{1-\text { loop }}, C_{f f^{\prime}}$ & no \\
\hline$\phi_{\mu, c, s}$ & no & no \\
\hline$\phi_{\tau, t, b}$ & no & $d_{u, d, e}^{2-l o o p}(\tilde{t}, \tilde{b}, \tilde{\tau}), \tilde{d}_{u, d}^{2-l o o p}(\tilde{t}, \tilde{b}, \tilde{\tau}), d^{3 \mathrm{G}}$ \\
\hline$\phi_{1,2}$ & $d_{u, d, e}^{1-l o o p}, \tilde{d}_{u, d}^{1-l o o p}, C_{f f^{\prime}}$ & $d_{u, d, e}^{2-l o o p}\left(\chi^{ \pm, 0}\right)$ \\
\hline$\phi_{3}$ & $d_{u, d}^{1-l o o p}, \tilde{d}_{u, d}^{1-l o o p}, C_{f f^{\prime}}$ & $d^{3 \mathrm{G}}$ \\
\hline
\end{tabular}

Table 1. Summary of how the CP-violating sources in MSSM generate various CP-odd operators at one-loop and two-loop level.

\begin{tabular}{|c|c|c|}
\hline$d_{n}$ & $d_{\mathrm{Tl}}$ & $d_{\mathrm{Hg}}$ \\
\hline$d_{u, d}, \tilde{d}_{u, d}, d^{3 G}, C_{f f^{\prime}}$ & $d_{e}, C_{f f^{\prime}}$ & $d_{e}, \tilde{d}_{u, d}, C_{f f^{\prime}}$ \\
\hline
\end{tabular}

Table 2. Summary of relevant CP-odd operators of neutron, Thallium, and Mercury atom EDMs.

(d) In the limit of heavy first and second generation sfermions, the "bino" phase $\phi_{1}$ is essentially unconstrained by present EDM bounds. A future neutron or electron EDM measurement with $\sim 100$ times better sensitivity would probe the impact of this phase at a level of interest for cosmology.

In the remainder of the paper, we organize the discussion of our analysis leading to the findings above as follows: In section 2, we give a general discussion about the CP-violating phase structure of the MSSM, we address how each phase impacts the various EDMs, and we outline the eneral setup of our analysis. In section 3 , we investigate in detail how each phase is constrained by current EDM bounds, we study the correlations between the various EDM bounds on the most strongly constrained phases, namely $\phi_{2}, \phi_{3}$, and $\phi_{u, d}$, and we discuss the phenomenology implications of the other loosely constrained phases. Finally, we devote section 4 to our summary and conclusions.

\section{CP-violating phases in MSSM and the setup for analysis}

The Minimal Supersymmetric Extension to the Standard Model of particle physics introduces a plethora of new and unknown parameters. Many of these parameters are connected to new sources of $\mathrm{CP}$ or flavor violation, or both. Although EDMs could, in principle, be induced by all CP-violating parameters including both flavor-conserving and flavor-violating ones, they are most sensitive to flavor-conserving CP-violating phases, including those associated with the bilinear coupling $b$ and Higgsino mass term $\mu$ in the Higgs-Higgsino sector, the soft-supersymmetry breaking Majorana masses $M_{1}, M_{2}$, and $M_{3}$ in the gaugino sector, and the trilinear couplings $A_{f}$ in the sfermion sector.

This notwithstanding, not all the new CP violating phases appearing in the MSSM are physical. In fact, there exist two transformations that can be employed to rotate away two phases [70]. We choose a convention where $\mu$ and $b$ are real, and the remaining phases mentioned above are all physical. In particular, the physical phases include the phases $\phi_{1,2,3}$ of the gaugino masses $M_{1,2,3}$, and the phases $\phi_{u, d, e}, \phi_{c, s, \mu}$, and $\phi_{t, b, \tau}$ of the sfermion 
trilinear couplings $A_{u, d, e}, A_{c, s, \mu}$, and $A_{t, b, \tau}$, respectively. As shown in table 2, these phases play different roles in generating various CP-odd operators, including the electron EDM $d_{e}$, quark EDM $d_{q}$ and Chromo-EDM $\tilde{d}_{q}$, the Weinberg 3-gluon operator $d^{3 G}$, and the 4-fermion CP-odd operator $C_{f f^{\prime} \cdot}{ }^{7}$ These CP-odd operators are responsible for the EDMs of the neutron, as well as of that of the Thallium and Mercury atoms, as summarized in table 2. In particular, in the MSSM the Thallium EDM is dominated by the electron EDM operator $d_{e}$, and possibly by the four-fermion operator $C_{f f^{\prime}}$ if $\tan \beta>30$ [59]; the neutron EDM, which we compute here using QCD sum rule results [62-65], mainly stems from the EDM and chromo-EDM operators of the $u$ and $d$ quarks, $d_{u, d}$ and $\tilde{d}_{u, d}$, and from the 3 -gluon term $d^{3 G}$; lastly, the Mercury EDM is generated primarily by the chromo-EDM operators $\tilde{d}_{u, d}[29]$. A combination of table 2 and table 2 provides information on how each CP-violating phase is constrained by which experimental EDM bound.

Among all contributions, some of the dominant ones stem from the one-loop induced EDM and Chromo-EDM operators $d_{e}, d_{u, d}$, and $\tilde{d}_{u, d}$. These contributions always involve the first-two generations of sleptons and squarks, and therefore are asymptotically suppressed in the limit where these scalar fermions are very heavy [71]. Obviously, the effect of the CP-violating phases $\phi_{u}, \phi_{d}, \phi_{s}, \phi_{c}, \phi_{e}, \phi_{\mu}$ from the first-two generations sfermions are completely suppressed in this situation, and would not show up in any other observable signature. In contrast, the effects of other phases, including $\phi_{1,2,3}$ in the gaugino sector and $\phi_{t}, \phi_{b}, \phi_{\tau}$ in the third-generation sfermion sector (thanks to larger Yukawa couplings), are not as strongly suppressed in the decoupling limit of heavy first and second generation sfermions, and they might induce interesting effects that could manifest themselves at colliders or in other experiments sensitive to $\mathrm{CP}$ violation.

With these considerations in mind, we study cases where the sfermion masses for the first two generations are either light or heavy. For each case, we explore in detail the massand $\tan \beta$-dependence of the EDM bounds on each individual phase. We choose a set of mass parameters corresponding to a light spectrum as the reference point (we call this "Case I"); we then study the effect on the constraints as the relevant mass scales increase (the limiting case is indicated as "Case II"). The reference values we choose for the relevant supersymmetric parameters $M_{1,2,3}, \mu, A_{f}$ of all flavors $f$, the charged Higgs mass $M_{H^{ \pm}}$, and the third generation sfermion masses $m_{L_{3}, R_{3}}$ in both cases are as follows:

$$
\begin{aligned}
\left|M_{1}\right|=150 \mathrm{GeV}, & \left|M_{2}\right|=250 \mathrm{GeV}, & \left|M_{3}\right|=550 \mathrm{GeV}, \\
|\mu|=225 \mathrm{GeV}, & \left|A_{f}\right|=175 \mathrm{GeV}, & M_{H^{ \pm}}=500 \mathrm{GeV}, \\
& m_{L_{3}}=m_{R_{3}}=200 \mathrm{GeV}, &
\end{aligned}
$$

We set the first-two generation sfermion masses, in the two cases, to:

$$
\begin{aligned}
& \text { CASE I }: m_{L_{1,2}}=m_{R_{1,2}}=200 \mathrm{GeV}, \\
& \text { CASE II }: m_{L_{1,2}}=m_{R_{1,2}}=10 \mathrm{TeV} .
\end{aligned}
$$

In the study of each phase, we look at how EDM constraints are affected by changes in the relevant mass scale, keeping all other masses set to their reference values.

\footnotetext{
${ }^{7}$ For the specific form of each of these operators, see, e.g. refs. [39].
} 

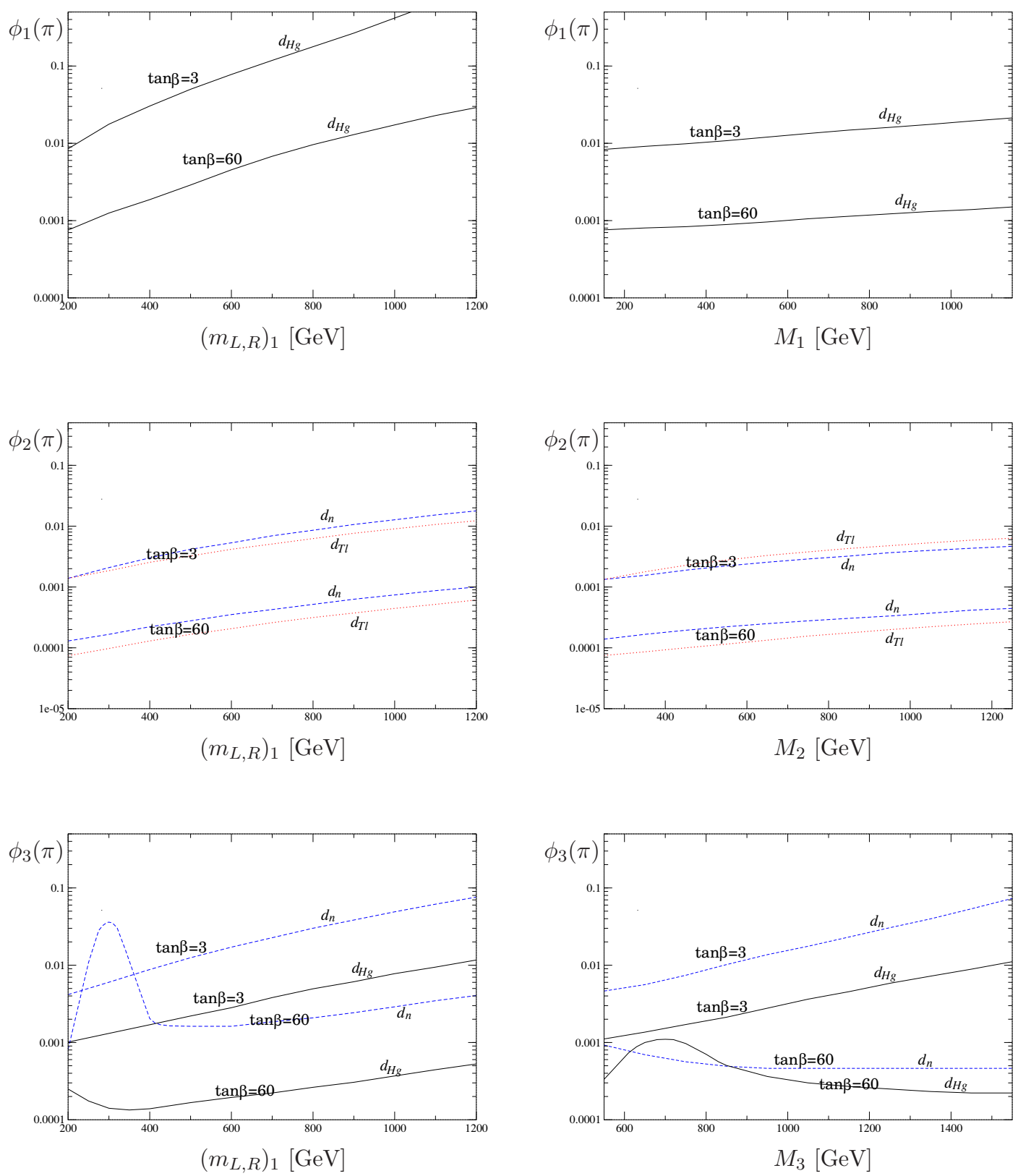

Figure 1. Constraints on the CP violating phases $\phi_{1}, \phi_{2}$ and $\phi_{3}$ (from top to bottom) versus $\left(m_{L, R}\right)_{1}$ (left panels) and versus $M_{1,2,3}$ (right panels) from experimental limits on the Mercury (black solid lines), Tallium (red dotted) and neutron (blue dashed) EDMs, in the case of light first-two generations of sfermions (case I, see eq. 2.2).

\section{Detailed analysis}

In the present study, we classify the MSSM CP-violating phases under consideration into two groups, 

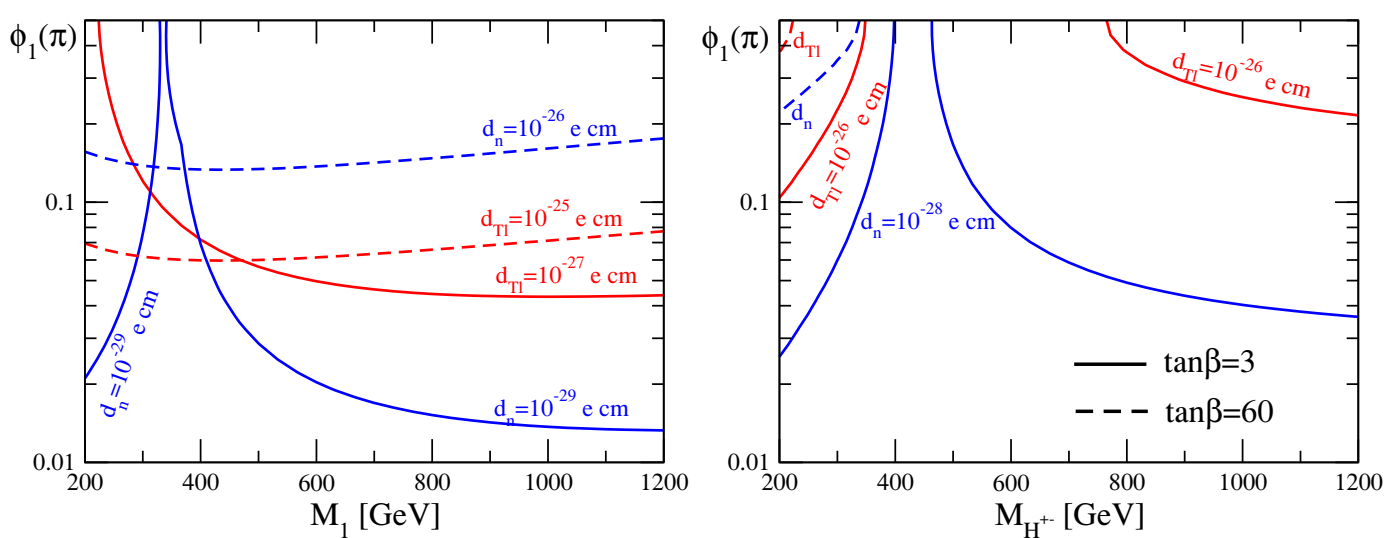

Figure 2. Curves of constant values for the Thallium (red) and neutron (blue) EDM as a function of $M_{1}$ and $M_{H^{ \pm}}$in the case of heavy first-two generations of sfermions (case $I I$ ) as in eq. 2.3. Because current EDM limits do not constrain $\phi_{1}$ in this case, curves correspond to representative future EDM sensitivities. Solid and dashed curves correspond, respectively, to $\tan \beta=3$ and 60 .
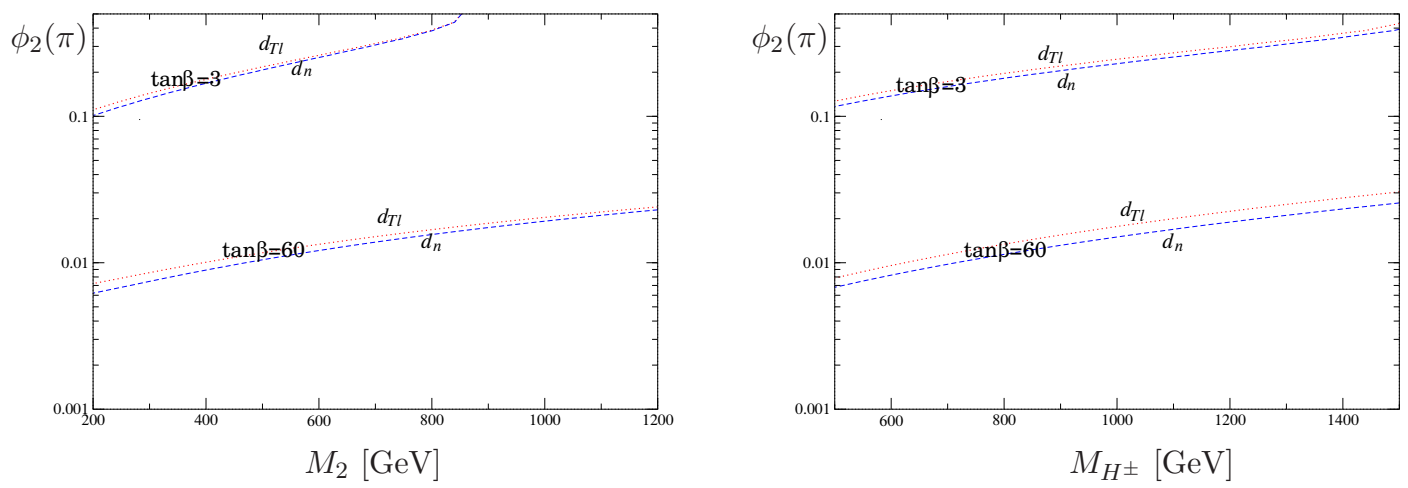

Figure 3. The $M_{2^{-}}$and $M_{H^{ \pm}}$- dependent constraints on $\phi_{2}$ from neutron and Thallium EDMs in the case of heavy first-two generations of sfermions (case $I I$ ) as in eq. 2.3.

(i) phases in the higgsino-gaugino sector: $\phi_{1}, \phi_{2}$, and $\phi_{3}$, and

(ii) phases in the sfermion sector: $\phi_{u}, \phi_{d}, \phi_{e}, \phi_{c}, \phi_{s}, \phi_{\mu}, \phi_{t}, \phi_{b}, \phi_{\tau}$,

and we study the two groups of phases individually.

\subsection{Phases in the higgsino-gaugino sector: $\phi_{1}, \phi_{2}$, and $\phi_{3}$}

These three phases contribute to the EDM and Chromo-EDM operators both at the oneand two-loop level; we therefore discuss here the constraints on them for both cases: (I) with light and (II) with heavy first-two sfermion generations.

Light sfermions. In the case where the first-two generation sfermions are light and one-loop EDMs and Chromo-EDMs are not suppressed, the phases $\phi_{1}$ and $\phi_{2}$ induce contributions to $d_{e}$, while $\phi_{1,2,3}$ generate $\left(d_{u, d}, \tilde{d}_{u, d}\right)$, and the four-fermion CP-odd operators 

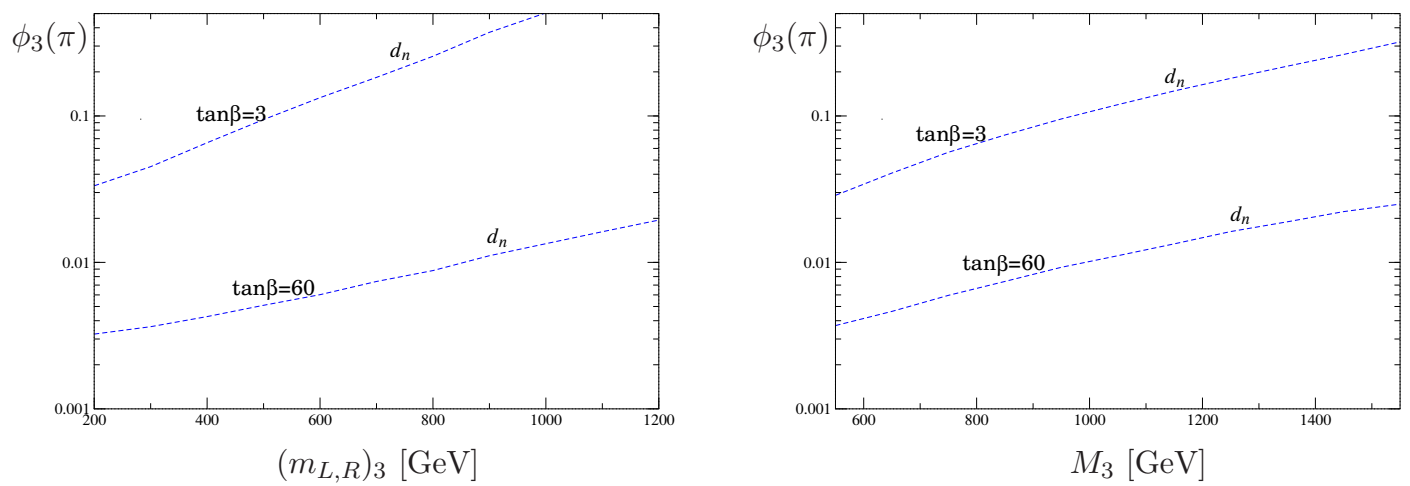

Figure 4. The $\left(m_{L, R}\right)_{3^{-}}$and $M_{3^{-}}$dependent constraints on $\phi_{3}$ from neutron EDMs in the case of heavy first-two generations of sfermions (case $I I$ ) as in eq. 2.3.

$C_{f f^{\prime}}$. In addition, $\phi_{3}$ also induces a non-zero contribution to the 3 -gluon operator $d^{3 G}$ at two-loop order [73]

We find that as far as the constraints on $\phi_{1}$ are concerned, the Mercury EDM, with its newest experimental bound, puts much more stringent bounds - namely by a factor of 10 or more on most of the parameter space we consider - than those from the neutron and Thallium EDM limits. We illustrate the resulting limits in figure 1, top panels. For the phase $\phi_{2}$, instead, the Mercury EDM puts less stringent limits, by a factor of a few, than the current Thallium and neutron EDM bounds, illustrated in figure 1, middle panels. We find that this is due to the cancellation of $\phi_{2}$ contributions to the electron EDM and quark chromo-EDMs that generate Mercury EDM. In fact, in a different mass region where the cancelation is not significant, the Mercury EDM constraint on $\phi_{2}$ may be comparable or even stronger than the current Thallium and neutron EDM bounds. For $\phi_{3}$, the new Mercury EDM bound puts a stronger constraint than the neutron EDM bound, while the current Thallium EDM bound is not stringent enough to put any constraint on $\phi_{3}$, due to suppressed contributions from $C_{f f^{\prime}}$ (see figure 1, lower panels).

Our results as a function of the relevant mass scales are summarized in figure 1 . In the upper penals, we show how the Mercury EDM constraint on $\phi_{1}$ depends on $\tan \beta$ (set to 3 and to 60 in the upper and lower curves, respectively) and on the relevant mass scales $\left(m_{L, R}\right)_{1}$ (left panel) and $M_{1}$ (right panel). The most important impact of $\phi_{1}$ on the Mercury EDM is through the neutralino one-loop contribution to the quark Chromo$\operatorname{EDM} \tilde{d}_{u, d}^{\chi^{0}}$, where the external gluon is only attached to squarks in the loop. In this case, the dependence on the sfermion and gaugino masses are somewhat non-trivial: The allowed values for $\phi_{1}$ grow quickly with increasing $\left(m_{L, R}\right)_{1}$ (left panel), but rather slowly with increasing $M_{1}$ (right panel). As $\left(m_{L, R}\right)_{1}$ increases to $1.2 \mathrm{TeV}, \phi_{1}$ can be as large as $\pi / 2(0.03 \pi)$ for $\tan \beta=3(60)$, indicating that a larger value for $\phi_{1}$ is allowed for smaller $\tan \beta$ when the one-loop contributions dominate.

In the two middle panels of figure 1, we show how the neutron (blue dashed lines) and the Thallium (red dotted lines) EDM constraints on $\phi_{2}$ depend on $\tan \beta$ and on the 
relevant mass scales $\left(m_{L, R}\right)_{1}$ and $M_{2}$. We notice that the current neutron and Thallium EDM bounds put comparable constraints on the wino phase $\phi_{2}$. The most important contributions from $\phi_{2}$ to the neutron and Thallium EDMs are through the chargino oneloop contribution to quark and electron EDMs $d_{u, d, e}^{\chi^{ \pm}}$, where the external photon is attached to both sfermions and charginos in the loop. In this case, the dependence on the sfermion and gaugino masses resemble the case of the neutralino loop. A comparison of the left and right panels shows that the allowed values of $\phi_{2}$ grows a little faster with increasing $\left(m_{L, R}\right)_{1}$ than with increasing $M_{2}$. For $\tan \beta=3(60)$, the phase $\phi_{2}$ is allowed to be within $10^{-2} \pi\left(0.6 \times 10^{-3} \pi\right)$ for $\left(m_{L, R}\right)_{1}$ up to $1.2 \mathrm{TeV}$, and $0.5 \times 10^{-2} \pi\left(0.3 \times 10^{-3} \pi\right)$ for $M_{2}$ up to $1.25 \mathrm{TeV}$.

In the lower panels of figure 1, we finally show how the constraints on $\phi_{3}$ depend on $\tan \beta$ and relevant mass scales $\left(m_{L, R}\right)_{1}$ (left) and $M_{3}$ (right). The black line indicates the Mercury EDM constraint, while the blue dashed line refers to the neutron EDM. We observe that the constraints on $\phi_{3}$ do not monotonically increase with mass scales, due to non-trivial cancelations among $\tilde{d}_{u, d}, d_{u, d}$, and $d^{3 G}$ in their contributions to the neutron EDM, and to cancellations among different contributions to $\tilde{d}_{u, d}$, which dominate the Mercury EDM. However, there is no common region where both the neutron and the Mercury EDM constraints are suppressed; hence, the cancellations among various $\phi_{3^{-}}$ dependent contributions never open a region in this portion of parameter space region where this CP-violating phase can be large, independent of the values of the other phases. For $\tan \beta=3(60)$, the phase $\phi_{3}$ is allowed to be within $10^{-2} \pi\left(0.5 \times 10^{-3} \pi\right)$ for $\left(m_{L, R}\right)_{1}$ up to $1.2 \mathrm{TeV}$, and $10^{-2} \pi\left(0.2 \times 10^{-3} \pi\right)$ for $M_{3}$ up to $1.55 \mathrm{TeV}$.

Heavy sfermions. In the case where the first-two generations of sfermions are heavy (case II), and therefore the one-loop EDMs and Chromo-EDMs are suppressed, the gaugino phases $\phi_{1}$ and $\phi_{2}$ still induce a non-vanishing 4-fermion CP-odd operator $C_{f f^{\prime}}$ due to loopinduced mixing between the CP-even and CP-odd Higgses that are exchanged between the fermions. In addition, $\phi_{1}$ and $\phi_{2}$ induce $d_{e}$ and $d_{u, d}$ at the two-loop level, while $\phi_{3}$ induces 3 -gluon operator $d^{3 G}$ and Chromo-EDM $\tilde{d}_{u, d}$ at the two-loop level.

Figure 2 shows that in case II (heavy sfermions) the phase $\phi_{1}$ is not constrained by current EDM bounds at all. This has important consequences for scenarios where the baryon asymmetry in the universe is generated via the mechanism of electroweak baryogenesis: a non-vanishing and large enough $\phi_{1}$ can generate the observed baryon asymmetry via the bino-driven scenario in the context of electroweak baryogenesis, even if $\phi_{2}=0$ [11]. In figure 2 , where we set $\phi_{2}=0$, we show contours of constant values for the neutron and Thallium EDMs that are below the current bounds, at $\tan \beta=3$ and 60 . Notice that, in contrast to the situation for $\tan \beta=60$, at $\tan \beta=3$ cancellations occur between the $W W$ and the $W^{ \pm} H^{\mp}$ contributions, the only non-vanishing graphs for $\phi_{2}=0$ [60]. In particular, ref. [60] pointed out that these two contributions have opposite signs, and if $\tan \beta$ has a value such that the two are comparable, then cancellations are possible, and indeed they occur for $M_{1} \sim 320 \mathrm{GeV}$ and for $m_{H} \sim 420 \mathrm{GeV}$ in our setup, as shown in figure 2. Similar cancellations do not take place at large $\tan \beta$, where the $W^{ \pm} H^{\mp}$ contribution dominates.

If instead the wino phase $\phi_{2}$ is non-vanishing (figure 3), two-loop contributions are much larger (see ref. [60] for a discussion on what makes wino-driven two-loop EDMs 

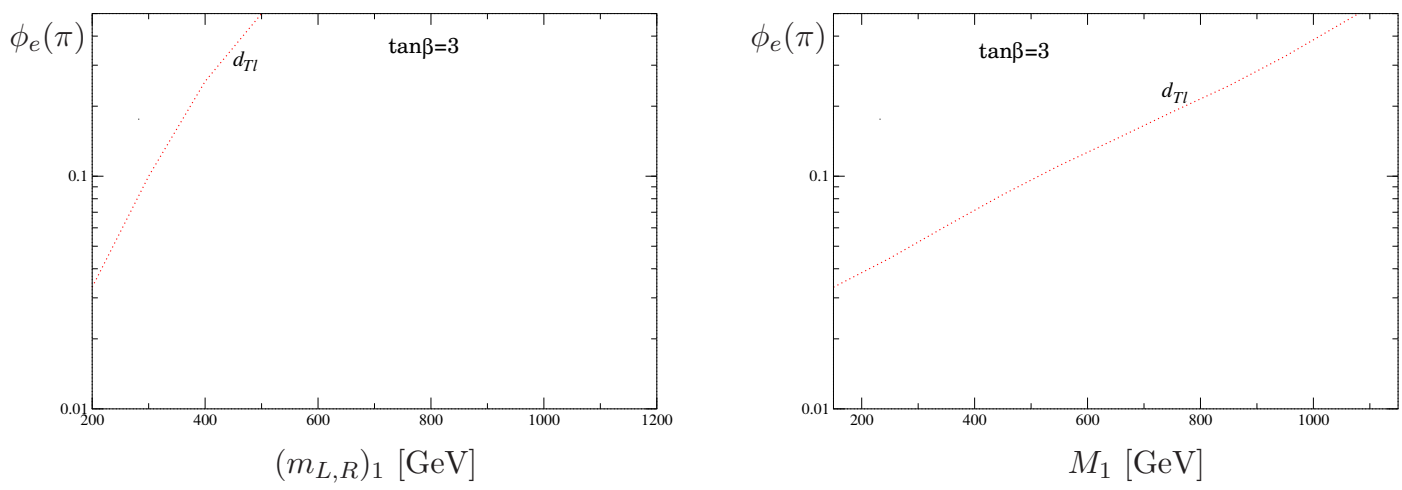

Figure 5. The $\left(m_{L, R}\right)_{1}$ - and $M_{1}$ - dependent constraints on $\phi_{e}$ from Thallium EDM in the case with light first-two generations of sfermions as in eq. 2.2. The $\tan \beta=3$ is used in making the plot. The constraints corresponding to other values of $\tan \beta$ are not shown, as the $\tan \beta$ dependence is found to be rather weak.

typically a factor of 50-100 larger than bino-driven ones). Specifically, with our choice of parameters, the limits on $\phi_{2}$ as a function of $M_{2}$ from the EDM bounds of Thallium atom and neutron range from $0.006 \pi$ for small $M_{2}=200 \mathrm{GeV}$ up to $0.02 \pi$ for $M_{2} \sim 1 \mathrm{TeV}$. A similar dependence is found for the second mass scale entering the two-loop contribution, namely the heavy Higgs sector, where we find that $\phi_{2}$ must be smaller than $0.007 \pi$ for small $m_{H^{ \pm}} \sim 500 \mathrm{GeV}$ and than $0.03 \pi$ for $m_{H^{ \pm}} \sim 1500 \mathrm{GeV}$.

In case II, the phase $\phi_{3}$ induces EDMs of neutron, Thallium, and Mercury. Since it does not induce an electron EDM $d_{e}$, its contribution to Thallium EDM is highly suppressed. The phase $\phi_{3}$ could induce sizable Mercury EDM through generating $\tilde{d}_{u, d}$ at two-loop. However, the most stringent constraint comes from the current neutron EDM bound. In figure 4 , we show how the constraint on $\phi_{3}$ from neutron EDM bound depends on $\tan \beta$ and relevant mass scales $\left(m_{L, R}\right)_{3}$ and $M_{3}$. We observe that for $\tan \beta=3(60)$, the $\phi_{3}$ is allowed to be as large as $\pi / 2(0.02 \pi)$ for $\left(m_{L, R}\right)_{1}$ as heavy as $1.2 \mathrm{TeV}$, and $0.3 \times \pi(0.02 \pi)$ for $M_{3} \leq 1.55 \mathrm{TeV}$.

\subsection{Phases in the sfermion sector: $\phi_{e}, \phi_{u}, \phi_{d}, \phi_{\mu}, \phi_{c}, \phi_{s}, \phi_{t}, \phi_{b}, \phi_{\tau}$}

We discuss here how CP violating phases in the sfermion sector are constrained generation by generation. The phases associated with the first sfermion generation, namely $\phi_{e}, \phi_{u}$ and $\phi_{d}$, yield significant contributions to the electron and $\mathrm{u}$ - and d-quark EDMs, as well as to the Chromo-EDM, all only at the one-loop level. Thus, we only discuss here the case where the first-two generations of sfermions are light (case I) as in the case where sfermions are heavy all of these contributions asymptotically vanish.

Besides contributions to the 4-fermion CP-odd operators $C_{f f^{\prime}}$ which are in general small, the phase $\phi_{e}$ contributes to the electron EDM, and is therefore constrained by the Thallium EDM. The phases $\phi_{u}$ and $\phi_{d}$ contribute to the EDM and to the Chromo-EDM of quarks, and are therefore constrained by both the neutron and the Mercury EDMs. The relevant mass scales in these one-loop contributions are $\left(m_{L, R}\right)_{1}$ and $M_{1,2,3}$. However, 

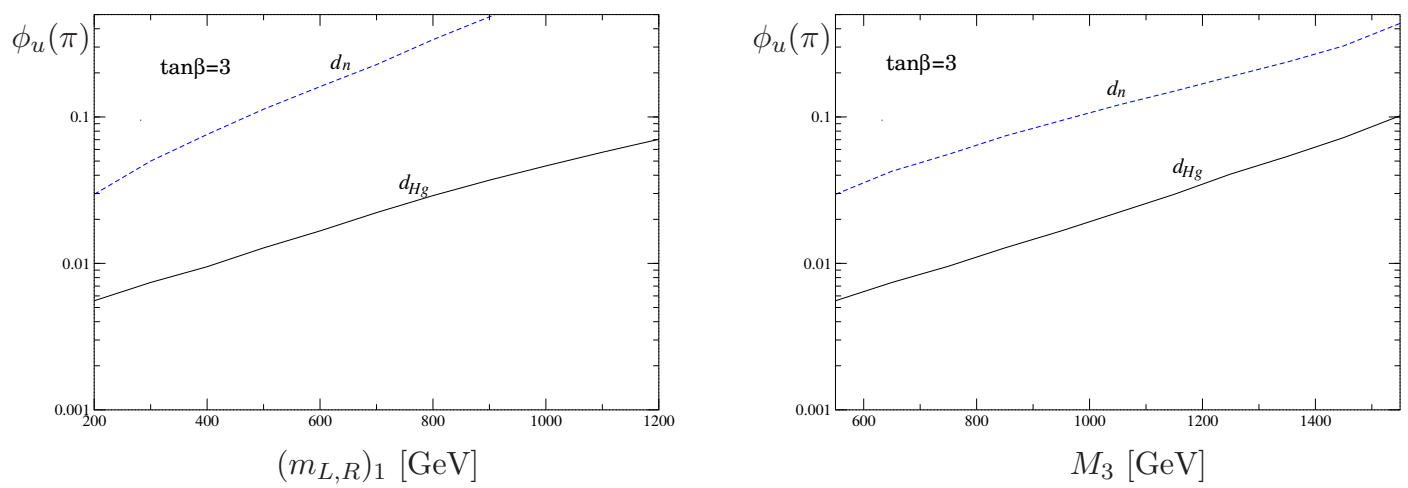

Figure 6. The $\left(m_{L, R}\right)_{1}$ - and $M_{3}$ - dependent constraints on $\phi_{u}$ from neutron and Mercury EDMs in the case with light first-two generations of sfermions as in eq. 2.2. The $\tan \beta=3$ is used in making the plot. The constraints corresponding to other values of $\tan \beta$ are not shown, as the $\tan \beta$ dependence is found to be rather weak.
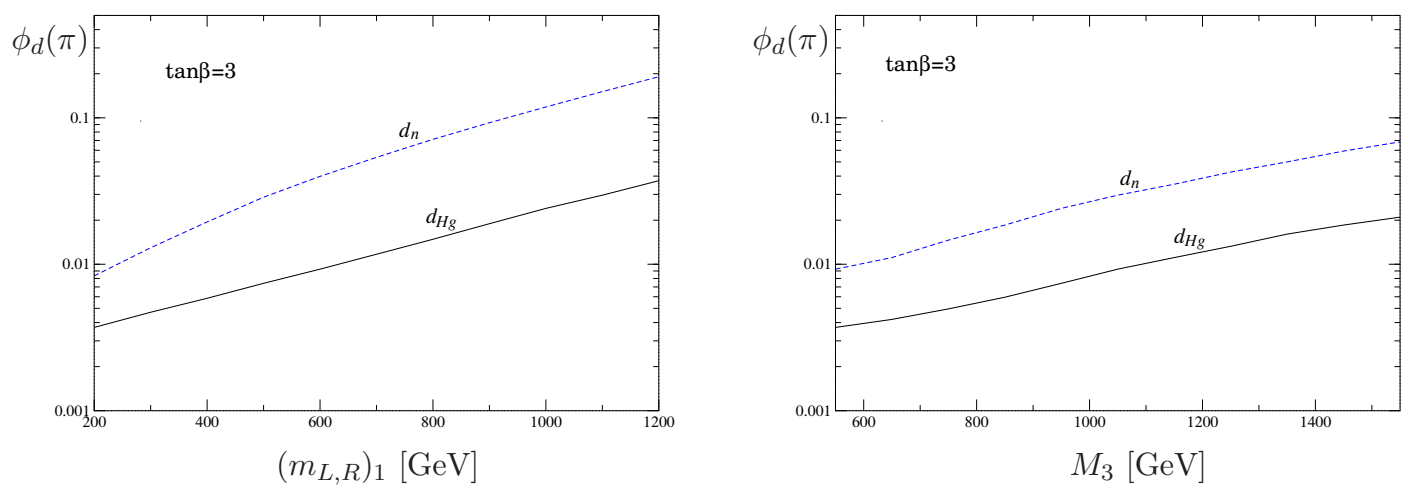

Figure 7. The $\left(m_{L, R}\right)_{1}$ - and $M_{3^{-}}$dependent constraints on $\phi_{d}$ from neutron and Mercury EDMs in the case with light first-two generations of sfermions as in eq. 2.2. The $\tan \beta=3$ is used in making the plot. The constraints corresponding to other values of $\tan \beta$ are not shown, as the $\tan \beta$ dependence is found to be rather weak.

since the one-loop contribution involving neutralinos dominates $d_{e}$, the constraint on $\phi_{e}$ depends mainly on $\left(m_{L, R}\right)_{1}$ and $M_{1}$. On the other hand, constraints on the squark CP violating phases $\phi_{u}$ and $\phi_{d}$ mainly depend on $\left(m_{L, R}\right)_{1}$ and $M_{3}$, since here the one-loop contribution involving gluinos dominates $d_{u, d}$ and $\tilde{d}_{u, d}$. The dependence on $\tan \beta$ is found to be rather weak.

As shown in figure 5, the allowed value of $\phi_{e}$ grows much faster with increasing $\left(m_{L, R}\right)_{1}$ than $M_{1}$, due to the dominant neutralino one-loop contribution. Constraints on this phase are rather weak and they reach $\pi / 2$ for $m_{L, R_{1}}$ of only $500 \mathrm{GeV}$, and for $M_{1}$ of $1.15 \mathrm{TeV}$.

As far as constraints on $\phi_{u}$ and $\phi_{d}$ are concerned, the new experimental limit on the Mercury EDM places a more stringent constraint (by a factor of a few) than the current neutron EDM bound. As shown in figures 6 and 7 , which display the $\left(m_{L, R}\right)_{1^{-}}$and $M_{3^{-}}$ 

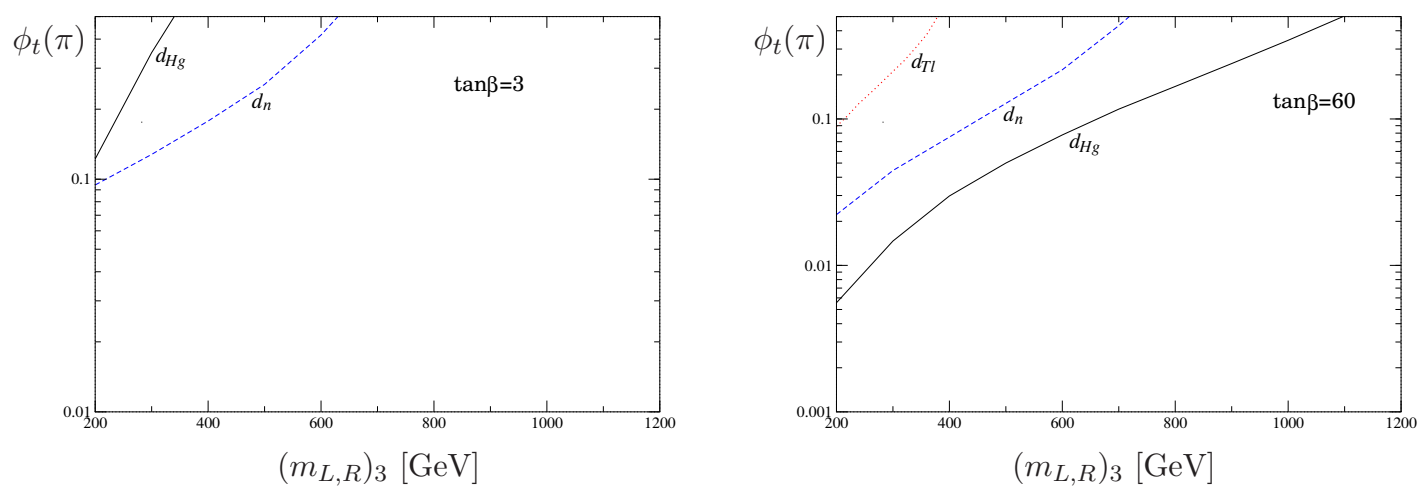

Figure 8. The $\left(m_{L, R}\right)_{3}$-dependent constraints on $\phi_{t}$ from neutron, Thallium, and Mercury EDMs.
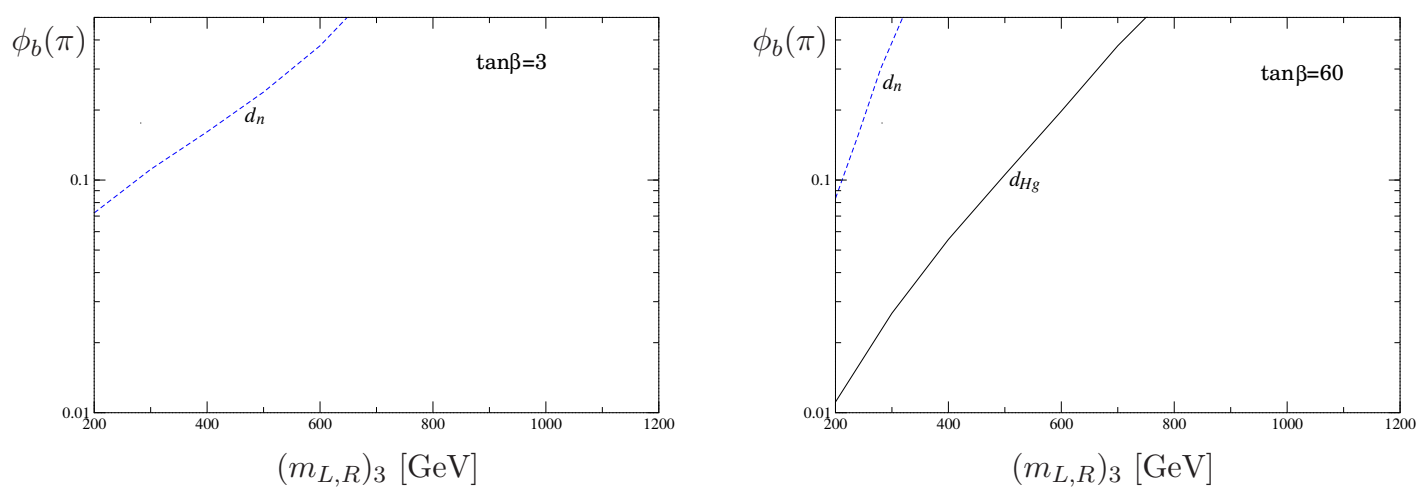

Figure 9. The $\left(m_{L, R}\right)_{3}$-dependent constraints on $\phi_{b}$ from neutron and Mercury EDMs.

dependent constraints on $\phi_{u}$ and $\phi_{d}$, respectively, the phase $\phi_{u}\left(\phi_{d}\right)$ is constrained to be $\leq 0.07 \pi(0.04 \pi)$ for $\left(m_{L, R}\right)_{1} \leq 1.2 \mathrm{TeV}$, and $0.1 \pi(0.02 \pi)$ for $M_{3} \leq 1.55 \mathrm{TeV}$.

The phases associated with the third generation sfermions $\phi_{t}, \phi_{b}$, and $\phi_{\tau}$ do not directly induce an electron or quark EDM, or a Chromo-EDM at the one-loop level, and therefore there is no difference here between the case with light and heavy first-two generations of sfermions. The third generation $\mathrm{CP}$ violating squark phases contribute to the electron and to the quark EDMs, Chromo-EDM s, 3-gluon operator, and 4-fermion operators at the two-loop level, and they all induce contributions to the EDMs of the neutron, Thallium and Mercury atoms. Our numerical study indicates that the current Thallium EDM bound is in general weaker than neutron and Mercury EDM bounds in constraining $\phi_{t}$, and does not put any constraint on $\phi_{b}$. The relative strength of the neutron and Mercury EDM bounds on $\phi_{t}$ and $\phi_{b}$ depends on $\tan \beta$, which drives the relative size of the Yukawa couplings of the bottom and top quarks. As shown in figure 8, at small $\tan \beta$, the neutron EDM constraint is stronger than that of the Mercury EDM, while at large $\tan \beta$, the Mercury EDM constraint becomes dominant. The $\phi_{t}$ and $\phi_{b}$ are rather loosely bounded and can reach $\pi / 2$ for $\left(m_{L, R}\right)_{3}$ of a few hundred $\mathrm{GeV}$. We find that the $\phi_{\tau}$ phase is not constrained 


\begin{tabular}{|c|c|c|c|c|c|c|}
\hline & \multicolumn{2}{|c|}{ CASE I } & \multicolumn{3}{|c|}{ CASE II } \\
\hline phases & $d_{\mathrm{Tl}}$ & $d_{n}$ & $d_{\mathrm{Hg}}$ & $d_{\mathrm{Tl}}$ & $d_{n}$ & $d_{\mathrm{Hg}}$ \\
\hline$\phi_{1}$ & weakly & weakly & weakly w. small $\tan \beta$ & not & not & not \\
\hline$\phi_{2}$ & strongly & strongly & strongly & weakly w. small tan $\beta$ & weakly w. small tan $\beta$ & not \\
\hline$\phi_{3}$ & not & strongly & strongly & not & weakly w. small tan $\beta$ & weakly \\
\hline$\phi_{e}$ & weakly & not & not & not & not & not \\
\hline$\phi_{u}$ & not & weakly & strongly & not & not & not \\
\hline$\phi_{d}$ & not & strongly & strongly & not & not & not \\
\hline$\phi_{\mu}$ & not & not & not & not & not & not \\
\hline$\phi_{c}$ & not & not & not & not & weakly & not \\
\hline$\phi_{s}$ & not & not & not & not & weakly \\
\hline$\phi_{t}$ & weakly & weakly & weakly & weakly & weakly \\
\hline$\phi_{b}$ & not & weakly & weakly & not & not & not \\
\hline$\phi_{\tau}$ & not & not & not & & &
\end{tabular}

Table 3. Summary of how the CP-violating phases in MSSM are constrained by current EDM bounds of neutron, Thallium, and Mercury atom, for both case I (with light first-two generations of sfermions as in eq. 2.2) and case II (with heavy first-two generations of sfermions as in eq. 2.3). We refer as "weakly constrained" if the phase can reach $\pi / 2$ with relevant mass scales within about $1 \mathrm{TeV}$, and "strongly constrained" otherwise.

by current EDM bounds at all.

Similarly, we find that the $\mathrm{CP}$ violating phases in the second generation of sfermions $\phi_{\mu}, \phi_{c}$, and $\phi_{s}$ are essentially unconstrained by experimental EDM limits.

\subsection{Correlated constraints on the most strongly constrained phases $\phi_{2}, \phi_{3}$ and $\phi_{u, d}$}

We summarize how each phase is constrained by current EDM bounds in table 3.2. The table shows that more than one phase is strongly constrained in case I (light first generation sfermions). These phases include $\phi_{2,3}$ in the higgsino-gaugino sector, and $\phi_{u, d}$ in the squark sector. In the following, we study the EDM constraints on the parameter space defined by pairs of such CP violating phases. For simplicity, we tie $\phi_{u, d}$ together, but keep $\phi_{2}$ and $\phi_{3}$ as independent. For each of the three combinations of phases $\left(\phi_{2}, \phi_{3}\right),\left(\phi_{2}, \phi_{u, d}\right)$, and $\left(\phi_{3}, \phi_{u, d}\right)$, we perform $\chi^{2}$ analysis and determine the region satisfying the combined neutron, Thallium, and Mercury EDM bounds at $95 \%$ c.l., for $\tan \beta=3$ and 60 , and $\left(m_{L, R}\right)_{1}=200,500$ and $1000 \mathrm{GeV}$.

As shown in figure 10, the allowed region for phases grows with the increase in mass scales in most cases. The only exception is the $\phi_{3}$ with large $\tan \beta$ (lower right panel of figure 10), which is due to the non-monotonic behavior shown in figure 1, lower panels.

The correlation between constraints on $\phi_{3}$ and $\phi_{u, d}$ (lower panels of figure 10) is the strongest, since both of them are dominantly constrained by the same bound, namely that from the Mercury EDM. On the other hand, the correlation between $\left(\phi_{2}, \phi_{3}\right)$ (figure 10 upper panels) and $\left(\phi_{2}, \phi_{u, d}\right)$ (figure 10 middle panels) are rather weak, because $\phi_{2}$ is constrained by different bounds - neutron and Thallium EDMs.

As a final illustration of the impact of the EDM constraints, we show in table 3.3 

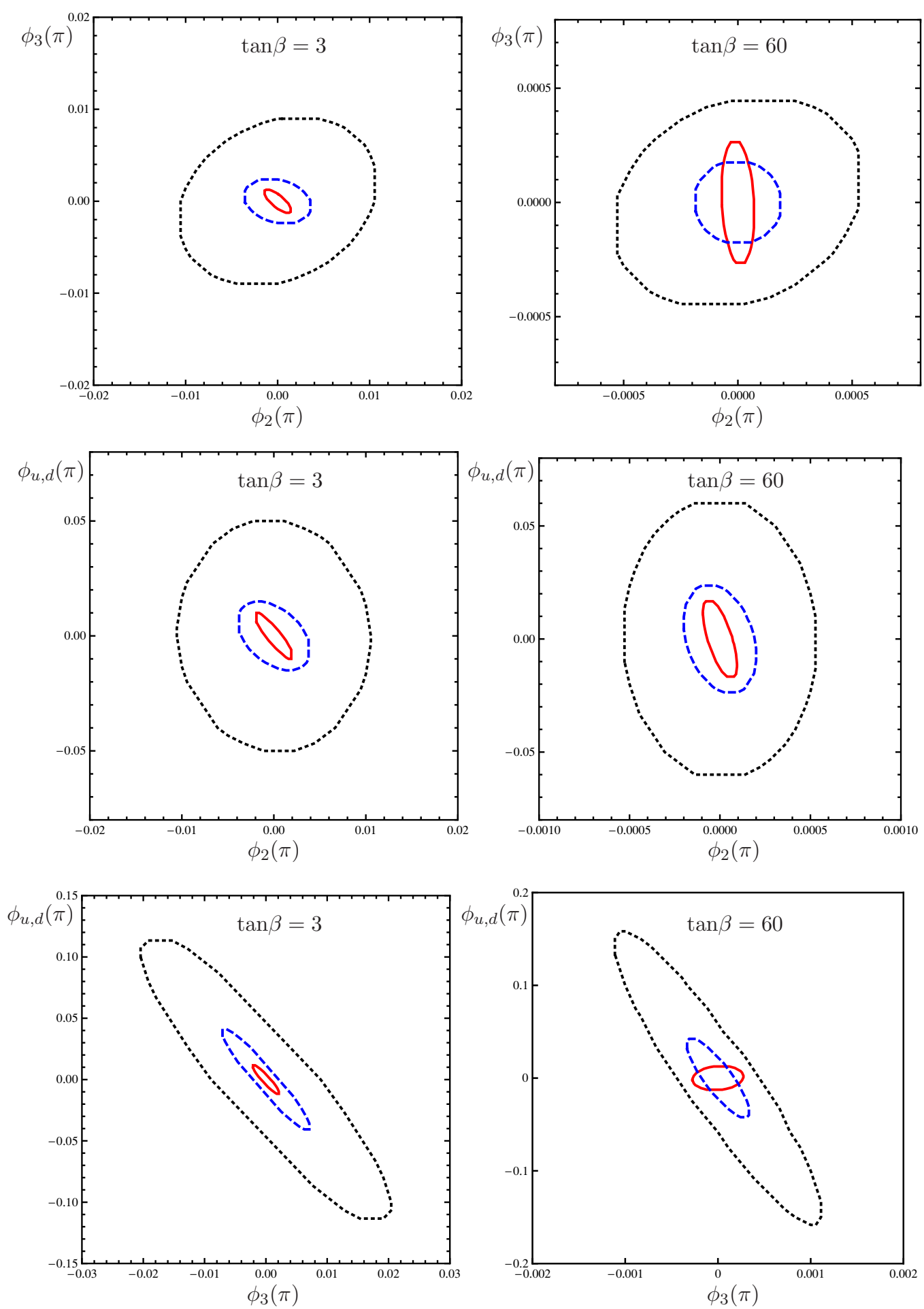

Figure 10. The correlated constraints on $\phi_{2}$ and $\phi_{3}$ (upper panels), $\phi_{u, d}$ (middle panels) and on $\phi_{3}$ and $\phi_{u, d}$ (lower panels), for $\tan \beta=3,60$ and $\left(m_{L, R}\right)_{1}=200 \mathrm{GeV}$ (red solid line), $500 \mathrm{GeV}$ (blue dashed line), and $1000 \mathrm{GeV}$ (black dotted line). Points inside the curve satisfy the $95 \%$ c.l. of the neutron, Thallium, and Mercury EDM bounds. 


\begin{tabular}{|c|c|c|c|c|c|c|}
\hline $\tan \beta$ & \multicolumn{3}{|c|}{3} & \multicolumn{3}{c|}{60} \\
\hline$\left(m_{L, R}\right)_{1}$ & $200 \mathrm{GeV}$ & $500 \mathrm{GeV}$ & $1000 \mathrm{GeV}$ & $200 \mathrm{GeV}$ & $500 \mathrm{GeV}$ & $1000 \mathrm{GeV}$ \\
\hline$\left|\phi_{2}\right|$ & $<2.1 \times 10^{-3}$ & $<5.0 \times 10^{-3}$ & $<1.5 \times 10^{-2}$ & $<9.3 \times 10^{-5}$ & $<2.5 \times 10^{-4}$ & $<6.9 \times 10^{-4}$ \\
\hline$\left|\phi_{3}\right|$ & $<2.8 \times 10^{-3}$ & $<9.7 \times 10^{-3}$ & $<2.8 \times 10^{-2}$ & $<3.1 \times 10^{-4}$ & $<4.2 \times 10^{-4}$ & $<1.5 \times 10^{-3}$ \\
\hline$\left|\phi_{u, d}\right|$ & $<1.8 \times 10^{-2}$ & $<6.0 \times 10^{-2}$ & $<0.17$ & $<1.7 \times 10^{-2}$ & $<5.6 \times 10^{-2}$ & $<0.21$ \\
\hline
\end{tabular}

Table 4. Summary of the combined bounds at $95 \%$ c.l. on three phases $\left(\phi_{2}, \phi_{3}, \phi_{u, d}\right)$ for $\tan \beta=3$, 60 and $\left(m_{L, R}\right)_{1}=200,500$, and $1000 \mathrm{GeV}$, using current experimental limits of neutron, Thallium, and Mercury EDMs as in eq. (1).

\begin{tabular}{|c|c|c|c|c|c|c|}
\hline $\tan \beta$ & \multicolumn{3}{|c|}{3} & \multicolumn{3}{c|}{60} \\
\hline$\left(m_{L, R}\right)_{1}$ & $200 \mathrm{GeV}$ & $500 \mathrm{GeV}$ & $1000 \mathrm{GeV}$ & $200 \mathrm{GeV}$ & $500 \mathrm{GeV}$ & $1000 \mathrm{GeV}$ \\
\hline$\left|\phi_{2}\right|$ & $<2.1 \times 10^{-3}$ & $<5.0 \times 10^{-3}$ & $<1.5 \times 10^{-2}$ & $<9.2 \times 10^{-5}$ & $<2.5 \times 10^{-4}$ & $<6.9 \times 10^{-4}$ \\
\hline$\left|\phi_{3}\right|$ & $<8.7 \times 10^{-3}$ & $<2.3 \times 10^{-2}$ & $<6.1 \times 10^{-2}$ & $<1.9 \times 10^{-3}$ & $<1.5 \times 10^{-3}$ & $<3.6 \times 10^{-3}$ \\
\hline$\left|\phi_{u, d}\right|$ & $<2.3 \times 10^{-2}$ & $<6.6 \times 10^{-2}$ & $<0.18$ & $<2.8 \times 10^{-2}$ & $<5.6 \times 10^{-2}$ & $<0.23$ \\
\hline
\end{tabular}

Table 5. Summary of the combined bounds at $95 \%$ c.l. on three phases $\left(\phi_{2}, \phi_{3}, \phi_{u, d}\right)$ for $\tan \beta=3$, 60 and $\left(m_{L, R}\right)_{1}=200,500$, and $1000 \mathrm{GeV}$, using current experimental limits of neutron, Thallium EDMs as in eq. (1), and previous limits of Mercury EDM $d_{\mathrm{Hg}}^{\text {previous }}=2 \times 10^{-28} e \mathrm{~cm}$ (95\% c. l.) [72].

\begin{tabular}{|c|c|c|c|c|c|c|}
\hline $\tan \beta$ & \multicolumn{3}{|c|}{3} & \multicolumn{3}{|c|}{60} \\
\hline$\left(m_{L, R}\right)_{1}$ & $200 \mathrm{GeV}$ & $500 \mathrm{GeV}$ & $1000 \mathrm{GeV}$ & $200 \mathrm{GeV}$ & $500 \mathrm{GeV}$ & $1000 \mathrm{GeV}$ \\
\hline$\left|\phi_{2}\right|$ & $<2.1 \times 10^{-3}$ & $<4.4 \times 10^{-3}$ & $<1.3 \times 10^{-2}$ & $<3.6 \times 10^{-5}$ & $<2.2 \times 10^{-4}$ & $<5.6 \times 10^{-4}$ \\
\hline$\left|\phi_{3}\right|$ & $<7.4 \times 10^{-4}$ & $<6.1 \times 10^{-3}$ & $<1.9 \times 10^{-2}$ & $<2.8 \times 10^{-4}$ & $<2.8 \times 10^{-4}$ & $<7.4 \times 10^{-4}$ \\
\hline$\left|\phi_{u, d}\right|$ & $<1.2 \times 10^{-2}$ & $<3.0 \times 10^{-2}$ & $<7.8 \times 10^{-2}$ & $<6.0 \times 10^{-3}$ & $<2.2 \times 10^{-2}$ & $<7.6 \times 10^{-2}$ \\
\hline
\end{tabular}

Table 6. Summary of the combined bounds at $95 \%$ c.l. on three phases $\left(\phi_{2}, \phi_{3}, \phi_{u, d}\right)$ for $\tan \beta=3$, 60 and $\left(m_{L, R}\right)_{1}=200,500$, and $1000 \mathrm{GeV}$, using current experimental limits of Thallium and Mercury EDMs as in eq. (1), and future possible improved neutron EDM limit $d_{\mathrm{n}}^{\text {future }}=2.9 \times$ $10^{-28} e \mathrm{~cm}(90 \%$ c. l.).

\begin{tabular}{|c|c|c|c|c|c|c|}
\hline $\tan \beta$ & \multicolumn{3}{|c|}{3} & \multicolumn{3}{|c|}{60} \\
\hline$\left(m_{L, R}\right)_{1}$ & $200 \mathrm{GeV}$ & $500 \mathrm{GeV}$ & $1000 \mathrm{GeV}$ & $200 \mathrm{GeV}$ & $500 \mathrm{GeV}$ & $1000 \mathrm{GeV}$ \\
\hline$\left|\phi_{2}\right|$ & $<2.1 \times 10^{-5}$ & $<5.2 \times 10^{-5}$ & $<1.7 \times 10^{-4}$ & $<9.3 \times 10^{-7}$ & $<2.5 \times 10^{-6}$ & $<7.6 \times 10^{-6}$ \\
\hline$\left|\phi_{3}\right|$ & $<2.8 \times 10^{-3}$ & $<7.8 \times 10^{-3}$ & $<2.2 \times 10^{-2}$ & $<3.1 \times 10^{-4}$ & $<3.8 \times 10^{-4}$ & $<1.2 \times 10^{-3}$ \\
\hline$\left|\phi_{u, d}\right|$ & $<1.5 \times 10^{-2}$ & $<4.6 \times 10^{-2}$ & $<0.13$ & $<1.5 \times 10^{-2}$ & $<5.0 \times 10^{-2}$ & $<0.19$ \\
\hline
\end{tabular}

Table 7. Summary of the combined bounds at $95 \%$ c.l. on three phases $\left(\phi_{2}, \phi_{3}, \phi_{u, d}\right)$ for $\tan \beta=3$, 60 and $\left(m_{L, R}\right)_{1}=200,500$, and $1000 \mathrm{GeV}$, using current experimental limits of neutron and Mercury EDMs as in eq. (1), and future possible improved Thallium EDM limit $d_{\mathrm{Tl}}^{\text {future }}=9.0 \times 10^{-27} e \mathrm{~cm}$ (90\% c. l.).

the combined bounds (at $95 \%$ c.l.) on all the three phases $\left(\phi_{2}, \phi_{3}, \phi_{u, d}\right)$ implied by the current EDM experimental limits. For purposes of comparison, we also show the impact of (a) the reduction in the Mercury EDM bound compared to the previous result $\left(d_{\mathrm{Hg}}^{\text {previous }}=2 \times 10^{-28} e \mathrm{~cm}(95 \%\right.$ c. 1.$\left.)\right) \quad($ see table 3.3$)$; (b) a neutron EDM bound that is 100 times more stringent than the present limit, keeping all other EDM limits as at present (3.3); and (c) a similar improvement in the Thallium limit, keeping the neutron and Mercury limits as at present (3.3). 
The bounds on phases $\left(\phi_{2}, \phi_{3}, \phi_{u, d}\right)$ from the combined 3-phase $\chi^{2}$ analysis, as shown in table 3.3, are in fact rather close to the bounds from the combined 2-phase $\chi^{2}$ analysis, for $\phi_{2}$ shown in the upper and middle panels of figure 10, and for $\phi_{3}$ and $\phi_{u, d}$ shown in the lower panel of figure 10, simply because a strong correlation only exists between $\phi_{3}$ and $\phi_{u, d}$, and because the inclusion of $\phi_{2}$ does not substantially alter this correlation.

Comparing table 3.3 and 3.3, we observe that the most significant impact of the recent update on Mercury EDM bound is on $\phi_{3}$, while there is no impact on $\phi_{2}$ and only small impact on $\phi_{u, d}$. This can be understood by looking at figure 1 (middle and lower panels), 6 , and 7 . The most stringent bounds on $\phi_{2}$ are from the Thallium and neutron EDMs. The most stringent bound on $\phi_{3}$ is from the current Mercury EDM, and the second most stringent bound from neutron EDM is many times looser. The most stringent bound on $\phi_{d}$ is from the Mercury EDM bound, but the neutron EDM bound is rather close.

In principle, one might expect that the impact of future neutron EDM experiments would be apparent when comparing table 3.3 and 3.3. Assuming the neutron EDM limit becomes 100 times tighter than current one, the limits on all phases $\phi_{2}, \phi_{3}$, and $\phi_{u, d}$, however, would only change to be, at most, a few times smaller. This is because as the neutron EDM bound becomes the most stringent one for $\phi_{2}, \phi_{3}$, and $\phi_{u, d}$, strong correlations among all of these three phases emerge, making the limits on all of them much larger than when the we consider limits on individual phases. This correlation arises from the presence of cancellations between various contributions associated with the different phases, a situation that has been noted previously in the literature (see, e.g., ref. [43]).

The correlations arising from this "cancellation mechanism" is most easily observed by considering the combined constraints on pairs of phases. To illustrate, we show in figure 11 , the correlated constraints on $\left(\phi_{2}, \phi_{3}\right),\left(\phi_{2}, \phi_{u, d}\right)$, and $\left(\phi_{3}, \phi_{u, d}\right)$, respectively, using a prospective future neutron EDM bound. Comparing with figure 10, we see that with a future neutron EDM bound that is 100 times tighter, the neutron EDM would become the dominant constraint. In such a case, the cancellation exists among all the phases $\phi_{2}, \phi_{3}$, and $\phi_{u, d}$, leading to a much narrower region for allowed phase values. We also note that the orientations of the $95 \%$ C.L. ellipses involving $\phi_{3}$ can differ from what appears in figure 10 since for the neutron, the constraints arise from the effects of both the quark EDMs and chromo-EDMs, in contrast to the situation for Mercury where the chromo-EDMs dominate. The relative importance of the $d_{q}$ and $\tilde{d}_{q}$ contributions to $d_{n}$ can change with $m_{L, R}$, leading to changes in the orientation of the ellipses with the value of these mass parameters.

To asses the impact of the future Thallium EDM experiments, we compare table 3.3 with 3.3. Assuming the future Thallium EDM bound is 100 times tighter than current one, the limit on $\phi_{2}$ would correspondingly shrink by approximately 100 times, while the limit on $\phi_{3}$ and $\phi_{u, d}$ would not change much, indicating a 100 times tighter Thallium EDM bound would not be more stringent than current neutron and Mercury EDM bounds on constraining $\phi_{3}$ and $\phi_{u, d}$. 

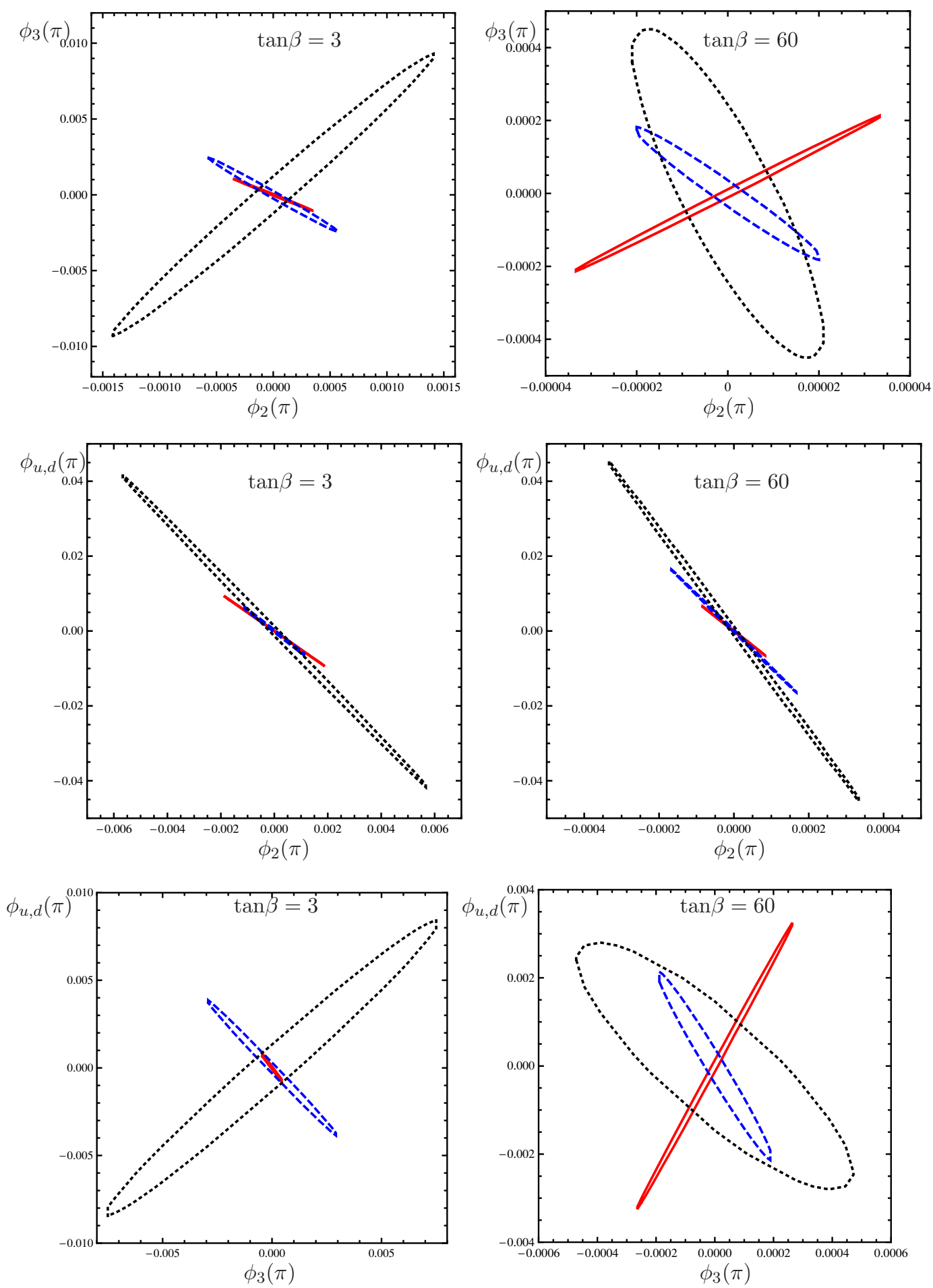

Figure 11. The correlated constraints on $\phi_{2}$ and $\phi_{3}$ (upper panels), $\phi_{u, d}$ (middle panels) and on $\phi_{3}$ and $\phi_{u, d}$ (lower panels), for $\tan \beta=3,60$ and $\left(m_{L, R}\right)_{1}=200 \mathrm{GeV}$ (red solid line), $500 \mathrm{GeV}$ (blue dashed line), and $1000 \mathrm{GeV}$ (black dotted line). Points inside the curve satisfy the $95 \%$ c.l. of the future neutron EDM bound, and current Thallium and Mercury EDM bounds. 


\subsection{Implication of loosely constrained phases on CP-violating and CP-conserv- ing phenomenologies}

As the EDM sensitivities improve, the impact of the most strongly constrained phases on other observables will be reduced. On the other hand, the effects of the more loosely constrained phases may still be apparent. In the higgsino-gaugino sector, for example, the only loosely constrained phase is $\phi_{1}$ (for relatively heavy first generation sfermions and small to moderate $\tan \beta$ ). This has important consequences in a rather wide range of phenomenological contexts, the most significant one being electroweak baryogenesis. A large $\phi_{1}$ can generate a sizeable contribution to the baryon asymmetry via bino-driven electroweak baryogenesis scenario, while the other scenario (wino-driven), which depends on $\phi_{2}$, is highly constrained by current EDM bounds [11].

Moreover, this phase is in general present in processes involving neutralinos both onshell and off-shell. Its effect shifts the neutralino mass spectrum and modify the couplings. Among CP-conserving quantities, it can lead to order one changes in the production rate, decay width, and branching ratios of neutralinos at colliders $[15,16]$. It also modifies the relic density, as well as direct and indirect detection rate of neutralino dark matter [32]. For example, as shown in ref. [32], the typical variations in the neutralino relic abundance from the $\phi_{1}$-dependence of couplings is about $\mathcal{O}(10-100 \%)$.

A potentially more direct probe of $\phi_{1}$ may be through its impact on $\mathrm{CP}$-violating observables at both the LHC and a future linear collider. At the LHC, it contributes to the triple product $\left(\vec{p}_{1} \times \vec{p}_{2}\right) \cdot \vec{p}_{3}$ associated with cascade decays of stops [12-14]

$$
g g \rightarrow \tilde{t}_{i} \tilde{t}_{i}, \quad \tilde{t}_{i} \rightarrow t \chi_{j}^{0}, \quad \chi_{j}^{0} \rightarrow \chi_{1}^{0} l^{+} l^{-},
$$

where the $\vec{p}_{i}$ are the momenta of final state charged particles in the decay chain of the stop. This observable is manifestly T-odd. If strong phases are negligible, then it can provide a probe of CPV. It is shown in ref. [13] that, assuming an order one phase, the signal can be detected with $10^{2}-10^{3}$ identified events. At a future linear collider, it may contribute to the triple product $\left(\vec{p}_{l^{+}} \times \vec{p}_{l^{-}}\right) \cdot \vec{p}_{e^{+}}$associated with neutralino production and subsequent leptonic decays [15-19]

$$
e^{+} e^{-} \rightarrow \chi_{1}^{0} \chi_{2}^{0}, \quad \chi_{2}^{0} \rightarrow \chi_{1}^{0} l^{+} l^{-},
$$

which is a genuine CP-odd observable. As shown in ref. [19], the CP asymmetry in this process can reach $10 \%$ for some values of the mass and phase parameters. It also contributes to another triple-product $\left(\vec{p}_{\tau} \times \vec{p}_{e^{+}}\right) \cdot \vec{s}_{\tau}$, a T-odd observable that is constructed using the transverse polarization $\vec{s}_{\tau}$ of the $\tau^{ \pm}$in the neutralino two-body decay

$$
e^{+} e^{-} \rightarrow \chi_{1}^{0} \chi_{2}^{0}, \quad \chi_{2}^{0} \rightarrow \tilde{\tau}^{ \pm} \tau^{\mp} .
$$

This correlation has been studied in ref. [20, 21], where it is shown that the corresponding asymmetry can reach values up to $60 \%$.

Finally, an off-shell neutralino in loop can in principle generate CP-odd observables in B-meson decays. However, the neutralino contributions is in most cases subdominant compared to other contributions involving gluions and charginos [22, 23]. 
Among all the loosely constrained phases $\phi_{e, \mu, \tau}$ in the slepton sector and $\phi_{c, s, t, b}$ in the squark sector, the implication of the third-generation phases $\phi_{\tau}$ and $\phi_{t, b}$ are most interesting. The phase $\phi_{\tau}$ contributes to the aforementioned T-odd observable $\left(\vec{p}_{\tau} \times \vec{p}_{e^{+}}\right) \cdot \vec{s}_{\tau}$ in the process outlined in eq. (3.3) [20,21]. The phase $\phi_{t, b}$ may generate sizable effect in Bmeson physics, even though they are flavor-conserving by themselves. The flavor-violation can be either from within the SM (CKM quark mixing), or from beyond the SM (offdiagonal elements in squark mass matrices). For example, a large $\phi_{t}$ can generate sizable deviation from SM for $S_{\phi\left(\eta^{\prime}\right) K_{S}}$, and generate large CP asymmetries $A_{\mathrm{CP}}(b \rightarrow s \gamma)$ in $b \rightarrow s \gamma$, through contributions involving charginos and charged-Higgs, where the flavor-violation comes from CKM mixing matrix [24, 25]. On the other hand, a deviation from SM for $S_{\phi K_{S}}$ can also be generated by chirality-flipping $L R$ and $R L$ gluino contributions [26], which can be induced by a flavor-violating chirality-conserving mass insertion $\left(\delta_{L L, R R}^{d}\right)_{23}$ and a chirality-flipping CP-violating mass insertion associated with the $\phi_{b}:\left(\delta_{L R, R L}^{d}\right)_{23}^{\text {induced }}=$ $\left(\delta_{L L, R R}^{d}\right)_{23} \times m_{b}\left(A_{b}-\mu \tan \beta\right) / \tilde{m}^{2}$.

The phases $\phi_{t, b}$ also generate mixing between CP-even and CP-odd Higgses in MSSM, changing their mass spectrum and couplings [34], which has important consequences for Higgs searches at the Tevatron and LHC [35]. As another example of their impact on CPconserving observables, the large $\phi_{t, b}$ also changes the neutralino annihilation and scattering cross section and thus are important for relic density, as well as for direct and indirect detection rates for neutralino dark matter [33].

\section{Conclusions}

In the present study, we analyzed the constraints from electric dipole moments on the size of $\mathrm{CP}$-violating phases in the MSSM, utilizing the dominant one- and two-loop contributions. We introduced the 2LEDM numerical code, interfaced to CPSuperH2.0, that encompasses all these contributions. We pointed out that not all CP violating phases in the MSSM are constrained to be small by null results from EDM searches. Our results are summarized for the ease of the reader in table 3.2. We differentiate there between a case where the first generation sfermions are light, and one-loop contributions to EDMs are significant, and one where they are heavy and one-loop contributions to EDMs are consequently suppressed.

We find that in the gaugino sector, the "wino" phase $\phi_{2}$ is fairly strongly constrained in both cases of light and heavy first generation sfermion masses $\left(m_{L, R}\right)_{1}$, the "gluino" phase $\phi_{3}$ is only strongly constrained in the case with light $\left(m_{L, R}\right)_{1}$, and the "bino" phase $\phi_{1}$ can be arbitrarily large in the case with heavy $\left(m_{L, R}\right)_{1}$, and can be sizable with light $\left(m_{L, R}\right)_{1}$ and small $\tan \beta$. We note that in earlier studies that employed a universality assumption $\left(\phi_{1}=\phi_{2}=\phi_{3}\right)$ this difference in EDM sensitivities to the different CPV phases in the gauge-Higgs sector of the MSSM was not apparent.

Turning to sfermion CP violating phases, only the stop CP violating phase $\phi_{t}$ (and only more weakly $\phi_{b}$ ) is marginally constrained in the limits of heavy first generation squarks and sleptons. For light first generation sfermions, rather stringent constraints arise for $\phi_{d}$ and for $\phi_{u}$ from the neutron and Mercury EDM bounds, and weak constraint for $\phi_{e}$ 
arises from the Thallium EDM limit. Virtually no constraint exists from EDMs on the CP violating phases for second and third generation leptons and for second generation squarks.

In summary, we showed in this paper that even in the absence of cancellations between contributions from different $\mathrm{CP}$ violating phases, null results on searches for the permanent EDM of the neutron and of atoms put constraints only on selected $\mathrm{CP}$ violating phases, leaving ample room for a rich phenomenology related to $\mathrm{CP}$ violation at colliders, $\mathrm{B}$ factories and dark matter searches.

\section{Acknowledgments}

M.J.R-M. thanks I. Rothstein for pointing out the recent CDF results. Y. Li thanks Xiangdong Ji for his hospitality at Shanghai Jiaotong University, where part of this work was completed S.P. is partly supported by an Outstanding Junior Investigator Award from the US Department of Energy (DoE), Office of Science, High Energy Physics, and by DoE Contract DE-FG02-04ER41268, NSF Grant PHY-0757911 and a Faculty Research Grant from the University of California, Santa Cruz. Y. Li and M.J.R-M. were supported in part by DoE contract DE-FG02-08ER41531 and by the Wisconsin Alumni Research Foundation.

Open Access. This article is distributed under the terms of the Creative Commons Attribution Noncommercial License which permits any noncommercial use, distribution, and reproduction in any medium, provided the original author(s) and source are credited.

\section{References}

[1] T. Ibrahim and P. Nath, CP violation from standard model to strings, Rev. Mod. Phys. 80 (2008) 577 [arXiv: 0705. 2008] [SPIRES].

[2] M.J. Ramsey-Musolf and S. Su, Low energy precision test of supersymmetry, Phys. Rept. 456 (2008) 1 [hep-ph/0612057] [SPIRES].

[3] P. Huet and A.E. Nelson, Electroweak baryogenesis in supersymmetric models, Phys. Rev. D 53 (1996) 4578 [hep-ph/9506477] [SPIRES].

[4] M.S. Carena, M. Quirós, A. Riotto, I. Vilja and C.E.M. Wagner, Electroweak baryogenesis and low energy supersymmetry, Nucl. Phys. B 503 (1997) 387 [hep-ph/9702409] [SPIRES].

[5] J.M. Cline, M. Joyce and K. Kainulainen, Supersymmetric electroweak baryogenesis, JHEP 07 (2000) 018 [hep-ph/0006119] [SPIRES].

[6] M.S. Carena, J.M. Moreno, M. Quirós, M. Seco and C.E.M. Wagner, Supersymmetric CP-violating currents and electroweak baryogenesis, Nucl. Phys. B 599 (2001) 158 [hep-ph/0011055] [SPIRES].

[7] C. Lee, V. Cirigliano and M.J. Ramsey-Musolf, Resonant relaxation in electroweak baryogenesis, Phys. Rev. D 71 (2005) 075010 [hep-ph/0412354] [SPIRES].

[8] C. Balázs, M.S. Carena, A. Menon, D.E. Morrissey and C.E.M. Wagner, The supersymmetric origin of matter, Phys. Rev. D 71 (2005) 075002 [hep-ph/0412264] [SPIRES].

[9] V. Cirigliano, S. Profumo and M.J. Ramsey-Musolf, Baryogenesis, electric dipole moments and dark matter in the MSSM, JHEP 07 (2006) 002 [hep-ph/0603246] [SPIRES]. 
[10] V. Cirigliano, Y. Li, S. Profumo and M.J. Ramsey-Musolf, MSSM baryogenesis and electric dipole moments: an update on the phenomenology, JHEP 01 (2010) 002 [arXiv:0910.4589] [SPIRES].

[11] Y. Li, S. Profumo and M. Ramsey-Musolf, Bino-driven electroweak baryogenesis with highly suppressed electric dipole moments, Phys. Lett. B 673 (2009) 95 [arXiv:0811.1987] [SPIRES].

[12] A. Bartl, E. Christova, K. Hohenwarter-Sodek and T. Kernreiter, Triple product correlations in top squark decays, Phys. Rev. D 70 (2004) 095007 [hep-ph/0409060] [SPIRES].

[13] P. Langacker, G. Paz, L.-T. Wang and I. Yavin, A T-odd observable sensitive to CP-violating phases in squark decay, JHEP 07 (2007) 055 [hep-ph/0702068] [SPIRES].

[14] J. Ellis, F. Moortgat, G. Moortgat-Pick, J.M. Smillie and J. Tattersall, Measurement of CP-violation in stop cascade decays at the LHC, Eur. Phys. J. C 60 (2009) 633 [arXiv: 0809.1607] [SPIRES].

[15] S.Y. Choi, H.S. Song and W.Y. Song, CP phases in correlated production and decay of neutralinos in the minimal supersymmetric standard model, Phys. Rev. D 61 (2000) 075004 [hep-ph/9907474] [SPIRES].

[16] V.D. Barger et al., CP-violating phases in SUSY, electric dipole moments and linear colliders, Phys. Rev. D 64 (2001) 056007 [hep-ph/0101106] [SPIRES].

[17] Y. Kizukuri and N. Oshimo, T odd asymmetry mediated by neutralino in $e^{+} e^{-}$annihilation, Phys. Lett. B 249 (1990) 449 [SPIRES].

[18] A. Bartl, H. Fraas, O. Kittel and W. Majerotto, CP asymmetries in neutralino production in $e^{+} e^{-}$collisions, Phys. Rev. D 69 (2004) 035007 [hep-ph/0308141] [SPIRES].

[19] A. Bartl, H. Fraas, S. Hesselbach, K. Hohenwarter-Sodek and G.A. Moortgat-Pick, A T-odd asymmetry in neutralino production and decay, JHEP 08 (2004) 038 [hep-ph/0406190] [SPIRES].

[20] A. Bartl, T. Kernreiter and O. Kittel, A CP asymmetry in $e^{+} e^{-} \rightarrow$ neutralino(i) neutralino $(j) \rightarrow$ neutralino $(j)$ tau stau $(k)$ with tau polarization, Phys. Lett. B 578 (2004) 341 [hep-ph/0309340] [SPIRES].

[21] S.Y. Choi, M. Drees, B. Gaissmaier and J. Song, Analysis of CP-violation in neutralino decays to tau sleptons, Phys. Rev. D 69 (2004) 035008 [hep-ph/0310284] [SPIRES].

[22] S. Bertolini, F. Borzumati, A. Masiero and G. Ridolfi, Effects of supergravity induced electroweak breaking on rare B decays and mixings, Nucl. Phys. B 353 (1991) 591 [SPIRES].

[23] F. Gabbiani, E. Gabrielli, A. Masiero and L. Silvestrini, A complete analysis of FCNC and $C P$ constraints in general SUSY extensions of the standard model, Nucl. Phys. B 477 (1996) 321 [hep-ph/9604387] [SPIRES].

[24] A. Bartl et al., General flavor blind MSSM and CP-violation, Phys. Rev. D 64 (2001) 076009 [hep-ph/0103324] [SPIRES].

[25] W. Altmannshofer, A.J. Buras and P. Paradisi, Low energy probes of CP-violation in a flavor blind MSSM, Phys. Lett. B 669 (2008) 239 [arXiv:0808.0707] [SPIRES].

[26] G.L. Kane et al., $B_{d} \rightarrow \phi K_{s} C P$ asymmetries as an important probe of supersymmetry, Phys. Rev. Lett. 90 (2003) 141803 [hep-ph/0304239] [SPIRES].

[27] D0 collaboration, V.M. Abazov et al., Evidence for an anomalous like-sign dimuon charge asymmetry, arXiv: 1005.2757 [SPIRES]. 
[28] L. Oakes, $\sin \left(2 \beta_{2}\right)$ at $C D F$ : updated measurements of the CP violating phase in the $B_{s}-\bar{B}_{s}$ system, talk given at Flavor Physics and CP Violation 2010, Mat 25-29, Torino, Italy (2010).

[29] M. Pospelov and A. Ritz, Electric dipole moments as probes of new physics, Annals Phys. 318 (2005) 119 [hep-ph/0504231] [SPIRES].

[30] T. Falk, A. Ferstl and K.A. Olive, New contributions to neutralino elastic cross-sections from CP-violating phases in the MSSM, Phys. Rev. D 59 (1999) 055009 [Erratum ibid. D 60 (1999) 119904] [hep-ph/9806413] [SPIRES].

[31] T. Falk, A. Ferstl and K.A. Olive, Variations of the neutralino elastic cross-section with CP-violating phases, Astropart. Phys. 13 (2000) 301 [hep-ph/9908311] [SPIRES].

[32] G. Bélanger, F. Boudjema, S. Kraml, A. Pukhov and A. Semenov, Relic density of neutralino dark matter in the MSSM with CP-violation, Phys. Rev. D 73 (2006) 115007 [hep-ph/0604150] [SPIRES].

[33] P. Gondolo and K. Freese, CP violating effects in neutralino scattering and annihilation, JHEP 07 (2002) 052 [hep-ph/9908390] [SPIRES].

[34] A. Pilaftsis and C.E.M. Wagner, Higgs bosons in the minimal supersymmetric standard model with explicit CP-violation, Nucl. Phys. B 553 (1999) 3 [hep-ph/9902371] [SPIRES].

[35] M.S. Carena, J.R. Ellis, S. Mrenna, A. Pilaftsis and C.E.M. Wagner, Collider probes of the MSSM Higgs sector with explicit CP-violation, Nucl. Phys. B 659 (2003) 145 [hep-ph/0211467] [SPIRES].

[36] C.A. Baker et al., An improved experimental limit on the electric dipole moment of the neutron, Phys. Rev. Lett. 97 (2006) 131801 [hep-ex/0602020] [SPIRES].

[37] B.C. Regan, E.D. Commins, C.J. Schmidt and D. DeMille, New limit on the electron electric dipole moment, Phys. Rev. Lett. 88 (2002) 071805 [SPIRES].

[38] W.C. Griffith et al., Improved limit on the permanent electric dipole moment of Hg-199, Phys. Rev. Lett. 102 (2009) 101601 [SPIRES].

[39] J.R. Ellis, J.S. Lee and A. Pilaftsis, Electric dipole moments in the MSSM reloaded, JHEP 10 (2008) 049 [arXiv:0808.1819] [SPIRES].

[40] T. Falk and K.A. Olive, Electric dipole moment constraints on phases in the constrained MSSM, Phys. Lett. B 375 (1996) 196 [hep-ph/9602299] [SPIRES].

[41] T. Ibrahim and P. Nath, The chromoelectric and purely gluonic operator contributions to the neutron electric dipole moment in $N=1$ supergravity, Phys. Lett. B 418 (1998) 98 [hep-ph/9707409] [SPIRES].

[42] T. Ibrahim and P. Nath, The neutron and the electron electric dipole moment in $N=1$ supergravity unification, Phys. Rev. D 57 (1998) 478 [Erratum ibid. D 58 (1998) 019901] [Erratum ibid. D 90 (1999) 079903] [hep-ph/9708456] [SPIRES].

[43] T. Ibrahim and P. Nath, The neutron and the lepton EDMs in MSSM, large CP-violating phases and the cancellation mechanism, Phys. Rev. D 58 (1998) 111301 [Erratum ibid. D 60 (1999) 099902] [hep-ph/9807501] [SPIRES].

[44] M. Brhlik, G.J. Good and G.L. Kane, Electric dipole moments do not require the CP-violating phases of supersymmetry to be small, Phys. Rev. D 59 (1999) 115004 [hep-ph/9810457] [SPIRES].

[45] T. Ibrahim and P. Nath, Corrections to the Higgs boson masses and mixings from chargino, $W$ and charged Higgs exchange loops and large CP phases, Phys. Rev. D 63 (2001) 035009 [hep-ph/0008237] [SPIRES]. 
[46] S. Abel, S. Khalil and O. Lebedev, EDM constraints in supersymmetric theories, Nucl. Phys. B 606 (2001) 151 [hep-ph/0103320] [SPIRES].

[47] L.E. Ibáñez and D. Lüst, Duality anomaly cancellation, minimal string unification and the effective low-energy Lagrangian of $4-D$ strings, Nucl. Phys. B 382 (1992) 305 [hep-th/9202046] [SPIRES].

[48] V.S. Kaplunovsky and J. Louis, Model independent analysis of soft terms in effective supergravity and in string theory, Phys. Lett. B 306 (1993) 269 [hep-th/9303040] [SPIRES].

[49] T. Kobayashi, D. Suematsu, K. Yamada and Y. Yamagishi, Nonuniversal soft scalar masses in superstring theories, Phys. Lett. B 348 (1995) 402 [hep-ph/9408322] [SPIRES].

[50] J.S. Lee et al., CPsuperH: a computational tool for Higgs phenomenology in the minimal supersymmetric standard model with explicit CP-violation, Comput. Phys. Commun. 156 (2004) 283 [hep-ph/0307377] [SPIRES].

[51] J.S. Lee, M. Carena, J. Ellis, A. Pilaftsis and C.E.M. Wagner, CPsuperH2.0: an improved computational tool for Higgs phenomenology in the MSSM with explicit CP-violation, Comput. Phys. Commun. 180 (2009) 312 [arXiv:0712.2360] [SPIRES].

[52] D. Chang, W.-Y. Keung and A. Pilaftsis, New two-loop contribution to electric dipole moment in supersymmetric theories, Phys. Rev. Lett. 82 (1999) 900 [Erratum ibid. 83 (1999) 3972] [hep-ph/9811202] [SPIRES].

[53] A. Pilaftsis, Higgs-boson two-loop contributions to electric dipole moments in the MSSM, Phys. Lett. B 471 (1999) 174 [hep-ph/9909485] [SPIRES].

[54] D. Chang, W.-F. Chang and W.-Y. Keung, New constraint from electric dipole moments on chargino baryogenesis in MSSM, Phys. Rev. D 66 (2002) 116008 [hep-ph/0205084] [SPIRES].

[55] A. Pilaftsis, Higgs-mediated electric dipole moments in the MSSM: an application to baryogenesis and Higgs searches, Nucl. Phys. B 644 (2002) 263 [hep-ph/0207277] [SPIRES].

[56] S. Weinberg, Larger Higgs exchange terms in the neutron electric dipole moment, Phys. Rev. Lett. 63 (1989) 2333 [SPIRES].

[57] D.A. Dicus, Neutron electric dipole moment from charged Higgs exchange, Phys. Rev. D 41 (1990) 999 [SPIRES].

[58] J. Dai, H. Dykstra, R.G. Leigh, S. Paban and D. Dicus, CP violation from three gluon operators in the supersymmetric standard model, Phys. Lett. B 237 (1990) 216 [Erratum ibid. B 242 (1990) 547] [SPIRES].

[59] D.A. Demir, O. Lebedev, K.A. Olive, M. Pospelov and A. Ritz, Electric dipole moments in the MSSM at large $\tan \beta$, Nucl. Phys. B 680 (2004) 339 [hep-ph/0311314] [SPIRES].

[60] Y. Li, S. Profumo and M. Ramsey-Musolf, Higgs-Higgsino-gaugino induced two loop electric dipole moments, Phys. Rev. D 78 (2008) 075009 [arXiv:0806.2693] [SPIRES].

[61] T. Hahn, S. Heinemeyer, W. Hollik, H. Rzehak and G. Weiglein, FeynHiggs: a program for the calculation of MSSM Higgs-boson observables - Version 2.6.5,

Comput. Phys. Commun. 180 (2009) 1426 [SPIRES].

[62] M. Pospelov and A. Ritz, Theta-induced electric dipole moment of the neutron via QCD sum rules, Phys. Rev. Lett. 83 (1999) 2526 [hep-ph/9904483] [SPIRES].

[63] M. Pospelov and A. Ritz, Theta vacua, QCD sum rules and the neutron electric dipole moment, Nucl. Phys. B 573 (2000) 177 [hep-ph/9908508] [SPIRES]. 
[64] M. Pospelov and A. Ritz, Neutron EDM from electric and chromoelectric dipole moments of quarks, Phys. Rev. D 63 (2001) 073015 [hep-ph/0010037] [SPIRES].

[65] D.A. Demir, M. Pospelov and A. Ritz, Hadronic EDMs, the Weinberg operator and light gluinos, Phys. Rev. D 67 (2003) 015007 [hep-ph/0208257] [SPIRES].

[66] K.A. Olive, M. Pospelov, A. Ritz and Y. Santoso, CP-odd phase correlations and electric dipole moments, Phys. Rev. D 72 (2005) 075001 [hep-ph/0506106] [SPIRES].

[67] V.F. Dmitriev and V.V. Flambaum, Relativistic corrections to the nuclear Schiff moment, Phys. Rev. C 71 (2005) 068501 [nucl-th/0503040] [SPIRES].

[68] J.H. de Jesus and J. Engel, Time-reversal-violating Schiff moment of ${ }^{1} 99 \mathrm{Hg}$, Phys. Rev. C 72 (2005) 045503 [nucl-th/0507031] [SPIRES].

[69] S. Ban, J. Dobaczewski, J. Engel and A. Shukla, Fully self-consistent calculations of nuclear Schiff moments, Phys. Rev. C 82 (2010) 015501 [arXiv: 1003. 2598] [SPIRES].

[70] D.J.H. Chung et al., The soft supersymmetry-breaking lagrangian: theory and applications, Phys. Rept. 407 (2005) 1 [hep-ph/0312378] [SPIRES].

[71] A.G. Cohen, D.B. Kaplan and A.E. Nelson, The more minimal supersymmetric standard model, Phys. Lett. B 388 (1996) 588 [hep-ph/9607394] [SPIRES].

[72] M.V. Romalis, W.C. Griffith and E.N. Fortson, A new limit on the permanent electric dipole moment of Hg-199, Phys. Rev. Lett. 86 (2001) 2505 [hep-ex/0012001] [SPIRES].

[73] J. Dai, H. Dykstra, R.G. Leigh, S. Paban and D. Dicus, CP violation from three gluon operators in the supersymmetric standard model, Phys. Lett. B 237 (1990) 216 [Erratum ibid. B 242 (1990) 547] [SPIRES]. 